CAE Working Paper \#03-10

Optimal Exploitation of Renewable Resources under Uncertainty and the Extinction of Species

by

Tapan Mitra

$\&$

Santanu Roy

August 2003 


\title{
Optimal Exploitation of Renewable Resources under Uncertainty and the Extinction of Species*
}

\author{
Tapan Mitra ${ }^{\dagger}$ and Santanu Roy ${ }^{\ddagger}$
}

August, 2003.

\begin{abstract}
Under a minimal set of assumptions, the paper identifies conditions on the transition function of a Markov process leading to the following three scenarios: extinction, conservation, and the existence of a safe standard of conservation. These conditions are used to obtain restrictions on a framework of optimal exploitation of a renewable resource, under which the above three scenarios would occur. The biological growth function is allowed to be non-concave, and is subject to a random environmental shock, thereby making the results suitable for applications in a wide variety of models in renewable resource management.

Journal of Economic Literature Classification Numbers: D90, O11, O41, Q32.

Key words: Renewable resources, extinction, safe standard of conservation, Markov process, transition function, optimal resource management, stochastic dynamic programming.
\end{abstract}

* Research on this paper was completed when the second author visited Cornell University in July, 2003. We thank the Center for Analytic Economics and the Department of Economics at Cornell University for making this research visit possible.

${ }^{\dagger}$ Department of Economics, Cornell University, Ithaca, NY 14853, Email: tm19@cornell.edu.

${ }^{\ddagger}$ Department of Economics, Southern Methodist University, 3300 Dyer St., Dallas, TX 75275-0496. 


\section{Introduction}

Extinction of biological species is an important ecological concern of the current age. Extinction is likely whenever a renewable resource is harvested persistently at a rate exceeding the level required to sustain its current stock. The economics of extinction relates the depletion of resources to economic incentives that affect harvesting. Traditionally, economists have related overexploitation of resources to failures of markets and property rights. However, even if such failures are corrected and society "manages" its resources $o p$ timally; the nature of intertemporal trade-offs between current and future welfare that a society is willing to make can lead to eventual extinction. It is important to understand the way in which intertemporal preferences of society and the biological growth of resources interact to determine the possibility of extinction and conservation for an optimally managed resource.

One important factor here is the sensitivity of biological growth to random environmental fluctuations and the fact that persistent adverse environmental shocks can severely deplete resource stocks. Such environmental uncertainty also affects the incentive to harvest a resource. The nature of human exploitation and its effect on the dynamics of biological populations when the growth process of species is subject to random environmental shocks is not very well understood. In this paper we undertake a systematic study of this issue, while keeping our framework of analysis fairly general, so that our results can be applied to a variety of models, which can be reduced to our framework.

We begin by considering a framework (in Section 2) in which the resource stock evolves stochastically according to a Markov process with a given transition law. While we do place some structure on the transition function to help us reach concrete results with respect to extinction and conservation scenarios, this structure is in a sense minimal. In particular, we allow for discontinuities in the transition function. The obvious application one has in mind is where the transition function is derived from a dynamic optimization problem, which seeks to maximize the expected discounted sum of returns from exploitation of the resource over an infinite horizon. However, the framework can also be applied to settings, where the harvest of the resource is obtained competitively by a demand and a supply function of the resource, the latter being determined by the cost of harvesting.

In the above framework, we identify conditions under which the following three scenarios occur: (i) the resource becomes extinct (with probability one) 
starting from all positive initial stocks; (ii) the resource is conserved (with probability one) from all positive initial stocks; (iii) there is a positive resource stock (called a safe standard of conservation) such that, starting from all higher stocks, the resource is conserved (with probability one). Further, these are the only scenarios that can arise in this framework in the long-run.

In particular, the condition that we specify for extinction from all initial stocks is very tight. It is obvious that if, independent of current level, the resource stock size is not sustainable even under the best environmental circumstance, then stocks must eventually decline to zero. We show that extinction occurs globally under a much weaker condition, namely, when the transition function is such that the resource stock size is not sustainable under the worst environmental shock. The possibility of sustainability or growth under better environmental shocks does not in any way alter the eventual destiny of species in such situations. Further, this is a necessary condition for global extinction in the sense that if there is a positive stock size from which the resource is sustained under the worst environmental shock, then that stock is a safe standard of conservation. The implication is that in a stochastic model, the possibility of extinction is almost entirely determined by the way in which resource stocks evolve under the worst environmental circumstance.

The relevant existing literature, where conditions for extinction or nonextinction are specified on the Markov transition function is relatively small. In a stochastic growth model, Mirman (1972) outlined sufficient conditions to rule out the possibility of extinction. Boylan (1976) pointed out that these conditions were contradictory. A revised set of conditions specified by Boylan (1979) are very restrictive in our setting because they require the next period's stock to be both increasing and concave in current stock. ${ }^{1}$ A more useful set of conditions on the transition function under which a stochastic process of the kind considered in our paper converges to zero with probability one is contained in Athreya (2002). All of these papers impose continuity and other conditions on the class of admissible transition functions that are more restrictive than in our framework.

In Sections 3 and 4, we explore a model of optimal resource management by using the methods of stochastic dynamic programming. The return func-

\footnotetext{
${ }^{1}$ In an economic model of optimal resource harvesting, concavity of the transition function can be ensured if the natural growth function as well as the optimal investment rule are shown to be concave functions of the current stock. However, concavity is an unlikely property of the optimal investment rule except in very special examples.
} 
tion in the optimization exercise (the net welfare function) depends on the harvest of the resource. We deliberately exclude conservation motives from directly entering the return function, so that we may distinguish cases in which the pursuit of "self-interest" does indeed lead to extinction from cases in which it does not. The biological production function and the optimal investment policy determine the transition function governing the stochastic evolution of the resource stock. We apply the results of Section 2 to this transition function to determine conditions on the primitives of the optimization problem, under which global extinction, global conservation and the existence of a safe standard of conservation will arise.

Beginning with Clark (1973), much of the analysis of the economics of optimal extinction and conservation of renewable resources has been carried out in deterministic models. The conventional wisdom from this literature suggests that stocks of an optimally managed resource ought to be bounded away from zero as long as the resource has an intrinsic growth rate ${ }^{2}$ exceeding the rate at which society discounts the future; extinction is optimal if the resource is less productive than the discount rate. ${ }^{3}$ However, when the natural growth of the resource is stochastic, comparing productivity of the resource to the discount rate is no longer sufficient to characterize the possibility of extinction. Indeed, optimal stocks may be arbitrarily close to zero no matter how productive the resource is. ${ }^{4}$

One of the salient features of the natural growth of many species is that the productivity or biological growth rate is low from small stocks, but it increases as the stock becomes larger, though eventually the growth rate diminishes as the environmental carrying capacity is approached. Therefore, the biological growth or production function in a model of optimal renewable resource management is typically non-concave (such as S-shaped). Our model of optimal resource management analyzed is, in fact, identical to a one-sector stochastic optimal growth model with non-concave production function. ${ }^{5}$ However, while economic growth models focus on the existence,

\footnotetext{
${ }^{2}$ The intrinsic growth rate refers to the productivity of the resource at zero.

${ }^{3}$ If the intrinsic growth rate of the resource is less than the discount rate, then extinction is optimal from small stocks. If the resource is globally less productive than the discount rate, global extinction is optimal. See, among others, Lewis and Schmalensee (1979), Majumdar and Mitra (1982, 1983), Dechert and Nishimura (1983) and Cropper (1988).

${ }^{4}$ In the literature on optimal stochastic growth, Mirman and Zilcha (1976) showed that optimal stocks may not be bounded away from zero even though the production function has infinite slope at zero (that is, the intrinsic growth rate is infinite).

${ }^{5}$ The literature on stochastic optimal growth (Brock and Mirman (1972)) typically
} 
uniqueness and stability of a non-trivial invariant distribution for capital stocks, our focus is on the phenomenon of extinction; that is, whether the stocks approach zero over time and the probability with which this event occurs. ${ }^{6}$

The existing literature on the problem of characterizing extinction and non-extinction in terms of verifiable properties of the primitives of the dynamic optimization problem (intertemporal preferences and the biological growth or production function), is rather small. In models of optimal stochastic growth, the possibility of extinction has been ruled out by imposing the condition that the slope of the production function be infinite at zero and also that there be a strictly positive probability mass on the "worst" realization of the random shock. ${ }^{7}$ For most biological species, the rate of natural growth is rather small when the stock depletes to a level close enough to zero $^{8}$ and the assumption of infinite growth rate at zero is not very well suited to our purpose. In this paper, we do not impose Inada conditions on the production function and the probability distribution of the random shock is non-atomic. We show that the net welfare from harvesting plays an important role in the conditions for ruling out extinction. In general, our conditions are much tighter than in the existing stochastic growth literature.

In the literature on renewable resource management under uncertainty ${ }^{9}$, there is no general analysis of conditions for extinction that can be verified from information about the natural growth of the resource and the net welfare from harvesting. Reed (1974) provides sufficient conditions for conservation in a model where an $(\mathrm{s}, \mathrm{S})$ investment policy is optimal. When there are no

assumes that the production function is concave. For an analysis of the problem of stochastic optimal growth in a framework that allows for a non-concave production function, see Majumdar, Mitra and Nyarko (1989).

${ }^{6}$ From the perspective of economic growth theory, our analysis is relevant to the question of existence and nature of poverty traps in a non-convex economy (even when it is on its first-best path). From a methodological standpoint, it is worth noting that in establishing global stability of invariant distributions, models of economic growth impose fairly strong conditions to ensure that the capital stocks are bounded away from zero. The conditions for avoidance of extinction in our analysis are significantly weaker and suggest that convergence results in stochastic growth models may be obtained for a wider class of production functions.

${ }^{7}$ See, among others, Brock and Mirman (1972), Mirman and Zilcha (1975) and Majumdar, Mitra and Nyarko (1989).

${ }^{8}$ See, for example, Clark (1990), and the references cited in his book.

${ }^{9}$ See, Lewis (1981) and Clark (1990). 
fixed costs, these conditions assume that welfare is linear in consumption and that the resource growth function is strictly concave. Specialized models of a similar kind (with specific parametric form) are analyzed by Lande, Engen and Saether (1994) and Alvarez and Shepp (1998). ${ }^{10}$ The linearity in consumption of the welfare function in these models implies that the conditions for conservation are solely determined by the productivity of the resource relative to the discount rate. As our analysis will establish, this does not hold under more general conditions when the net welfare function is non-linear. A more general analysis of the conditions for conservation in a model where the utility function depends on both consumption as well as resource stock is contained in Olson and Roy (2000); the condition for conservation provided in our model can be viewed as special case of that paper when the marginal utility from resource stock is zero. However, there is no analysis of conditions for extinction in that paper.

From a technical point of view, our analysis is rendered difficult because of the simultaneous presence of uncertainty (the random environmental shocks) and non-concavities in the production (or biological growth) function of the resource. Results for the corresponding non-stochastic model, as well as the corresponding stochastic model with concave production function, are compared to our results at various points in Section 4.

All proofs are collected in the final section (Section 5).

\section{General Results on Conservation and Ex- tinction}

In this section, we characterize the possibilities of extinction and conservation of a renewable resource, whose evolution over time is governed by a given time stationary law of motion. This law of motion is summarized by a transition function defining the relationship between resource stocks in any two consecutive time periods.

We can think of the transition function as being a composition of two time-invariant elements: (i) a biological production function which determines the relationship between the size of the current stock left after harvest and the stock available in the next period; and (ii) a rule which determines

\footnotetext{
${ }^{10}$ Alvarez (1998) analyzes possibility of immediate extinction in a framework where eventual extinction occurs almost surely even if the resource is not harvested.
} 
the size of the stock which is left (after harvest) for reproduction and growth, given the current stock, which we refer to as an investment function. While the first element reflects a "technology" determined by nature, the second element is determined by human beings exploiting the resource.

The second element can be determined in various ways, depending on how human behavior is modeled. In the next section, we will follow standard practice and model this human behavior as arising from the solution of a stochastic dynamic optimization problem, in which the expected discounted sum of utilities is maximized subject to feasibility constraints. This will allow us to see the influence of the various parameters of the dynamic optimization problem on the nature of the investment function, and therefore the transition law, and help us to evaluate the role of these parameters in alternative extinction/conservation scenarios for the renewable resource.

In this section, however, we are not concerned about how human behavior is modeled. We wish to focus here purely on the relationship between the nature of a given transition function governing the evolution of a renewable resource, and the probability of survival or extinction of that resource.

\subsection{The Transition Function}

The arguments of the transition function are the stock of the resource and a random shock to the environment. We model the latter in the following way. We will suppose that the random shock can be represented by a real number in the interval $I=[a, b]$, where $0<a<b<\infty$. The law governing this shock (initially) will be represented by a distribution, $\mu$, with support $[a, b]$.

One needs to specify how shocks in the future will be related to this initial shock; we will make the fairly standard assumption that the shocks are independent and identically distributed. To do this formally, let $\Omega$ be the space of all infinite sequences $\left(\omega_{1}, \omega_{2}, \ldots\right)$ where $\omega_{t} \in[a, b]$ for $t \in \mathbb{N}$. Denote by $\mathcal{B}$ the collection of Borel subsets of $[a, b]$. Let $\mathcal{F}$ be the $\sigma$-algebra generated by cylinder sets of the form $\prod_{n=1}^{\infty} A_{n}$, where $A_{n} \in \mathcal{B}$ for all $n \in \mathbb{N}$, and $A_{n}=I$ for all but a finite number of values of $n$. For each $t \in \mathbb{N}$, denote by $\mathcal{F}_{t}$ the $\sigma$-algebra generated by cylinder sets of the form $\prod_{n=1}^{\infty} A_{n}$, where $A_{n} \in \mathcal{B}$ for all $n \in \mathbb{N}$, and $A_{n}=I$ for all $n \geq t+1$. Let $P$ be the product measure over $\mathcal{F}$ generated by the probability distribution $\mu$ over $[a, b]$. This defines a probability space $(\Omega, \mathcal{F}, P)$. Next, define the projection $r_{t}(\omega)=\omega_{t}$ for $t \in \mathbb{N}$. Then $\left\{r_{t}\right\}_{1}^{\infty}$ is a sequence of independent and identically distributed random variables on $(\Omega, \mathcal{F}, P)$. 
The stock of the resource will be the biomass of the particular species whose evolution we are studying. It will be represented by a non-negative real number, $y$. Thus, $Y \equiv \mathbb{R}_{+}$will be the space of the resource stock.

The transition function is a function $G: Y \times I \rightarrow Y$; thus, $G(y, r)$ defines the stock next period, when the current period stock is $y$, and $r$ is the realization of the environmental shock. The following assumptions on $G$ will be used in this section:

(G.1) $G(0, r)=0$ for all $r \in I$.

(G.2) $\alpha \equiv \lim _{y \rightarrow \infty}[G(y, b) / y]$ exists, and $\alpha<1$.

(G.3) $G$ is non-decreasing in $y$ and $r$.

In studying extinction and survival issues, (G.1) is a natural assumption to make. Assumption (G.2) reflects the fact that there is a maximum carrying capacity of the ecosystem, so that very high resource stocks cannot be sustained. Note that (G.2) implies that $G(y, b)<b$ for all $y$ large enough. We define:

$$
K=\inf \{z \in Y: G(y, b)<y \text { for all } y \in(z, \infty)\}
$$

Then, we have $G(y, b)<y$ for all $y \in(K, \infty)$.

Assumption (G.3) requires that for any specific realization of the random shock, the stock next period is non-decreasing in the size of the current stock. The transition function satisfies this restriction if the production function is non-decreasing in the size of the investment, and the investment function is non-decreasing in the current stock. The latter is a behavioral property found in a wide class of bio-economic models of resource extraction. Further, (G.3) requires that $G(\cdot, r)$ is weakly ordered according to $r$.

Associated with $G$ are two functions, $m: Y \rightarrow Y$ and $M: Y \rightarrow Y$, defined by:

$$
\left.\begin{array}{l}
m(y)=G(y, a) \text { for } y \in Y \\
M(y)=G(y, b) \text { for } y \in Y
\end{array}\right\}
$$

Given assumption (G.3), it is legitimate to refer to $M$ as the best transition function and to $m$ as the worst transition function.

In contrast to the above assumptions on the transition function, which are fairly standard, we need to impose the following technical condition on the transition function for our subsequent analysis (which is not so standard):

(G.4) For any $y>0, \mu\{r \in I: G(y, r)>G(y, a)\}=1$. Further, for every $y^{1}, y^{2}$, satisfying $0<y^{1}<y^{2}<\infty$,

$$
\sup _{y \in\left[y^{1}, y^{2}\right]}[G(y, r)-G(y, a)] \rightarrow 0 \text { as } r \downarrow a
$$


Assumption (G.4) imposes two restrictions. First, from any positive stock $y$, the event that the stock next period is exactly equal to that corresponding to the worst transition $G(y, a)$, has zero probability. This would always be satisfied if $G(y, r)$ is strictly increasing in $r$ for each $y>0$, and $\mu$ is absolutely continuous with positive density on $[a, b]$. Second, it requires that as $r$ decreases to $a$, the functions $G(y, r)$ converge to $G(y, a)$ uniformly in $y$ on any positive closed interval of stocks. If there is a function, $g: Y \rightarrow Y$, such that $G(y, r)=r g(y)$ for all $(y, r) \in Y \times I$ (that is, the random shock is multiplicative), then $g$ is non-decreasing in $y$ (by (G.3)), and so:

$$
\sup _{y \in\left[y^{1}, y^{2}\right]}[G(y, r)-G(y, a)]=(r-a) g\left(y^{2}\right) \rightarrow 0 \text { as } r \downarrow a
$$

We state an immediate implication of (G.4), which will be useful in our subsequent analysis.

Lemma 1 Let $0<p<p^{\prime}<\infty$ be given, and suppose that:

$$
d \equiv \sup \left\{G(y, a) / y: y \in\left[p, p^{\prime}\right]\right\}<1
$$

Then there exist $\lambda \in(0,(b-a))$ and $\theta \in(0,1)$ such that:

$$
G(y, r)<\theta y \text { for all } r \in[a, a+\lambda] \text { and all } y \in\left[p, p^{\prime}\right]
$$

Let us note that we do not require the transition function to be continuous in $y$. Even if the production function is continuous, there are many circumstances in which the investment rule need not be continuous in the stock level. ${ }^{11}$ Also, observe that we do not put any restriction on the slope of the transition function at zero stock (other than requiring it to be non-negative).

The transition function $G$ defines a stochastic process, which is the principal object of our study. Given any initial stock $y>0$, we will be concerned with the stochastic process $\left\{y_{t}(y, \omega)\right\}$, where $y_{t}(y, \omega)$ is $\mathcal{F}_{t}$ measurable for all $t \in \mathbb{N}$, and :

$$
\left.\begin{array}{l}
\text { (i) } y_{1}(y, \omega)=G\left(y, \omega_{1}\right) \\
(i i) y_{t+1}(y, \omega)=G\left(y_{t}(y, \omega), \omega_{t+1}\right) \text { for } t \in \mathbb{N}
\end{array}\right\}
$$

\footnotetext{
${ }^{11}$ In particular, in the non-convex dynamic optimization problem which we analyze in the next section, the optimal dynamic investment rule is not necessarily continuous in the current stock.
} 


\subsection{Concepts of Extinction and Conservation}

Extinction needs to be defined in a way so as to encompass the event that the resource stock is reduced to zero in finite time as well as the event that the stocks, while never being actually reduced to zero, become arbitrarily small over time. The latter possibility can occur in two ways. First, the stocks may actually converge to zero over time. Second, the stocks may get arbitrarily close to zero but need not actually converge to a limit stock. We adopt the second concept of extinction, which clearly has broader scope.

Formally, extinction is said to occur from an initial stock $y>0$, given a realization $\omega \in \Omega$, if:

$$
\liminf \inf _{t \geq 0} y_{t}(y, \omega)=0
$$

Conservation is said to occur from an initial stock $y>0$, given a realization $\omega \in \Omega$ if:

$$
\liminf \inf _{t \geq 0} y_{t}(y, \omega)>0
$$

Since our concept of extinction is broad, the corresponding concept of conservation (which is defined as the complement of extinction) is strong, requiring resource stocks to be bounded away from zero.

Both concepts are defined given a realization of a sequence of random shocks. A natural object of interest is the probability of the set of realizations of the sequence of random shocks for which extinction or conservation occur, starting from a given stock of the resource. This leads to the following definitions.

Extinction is said to occur from an initial stock $y>0$ if:

$$
P\left\{\omega \in \Omega: \liminf _{t \geq 0} y_{t}(y, \omega)=0\right\}=1
$$

Similarly, conservation is said to occur from an initial stock $y>0$ if:

$$
P\left\{\omega \in \Omega: \lim \inf _{t \geq 0} y_{t}(y, \omega)>0\right\}=1
$$

Note that these are strong definitions of extinction (conservation), requring that the event of extinction (conservation) possibilities occurs almost surely. Thus, logically, it would appear that there are many intermediate cases, in which the event of extinction (conservation) possibilities has a positive probability less than one. We will see that the actual set of intermediate cases that the model generates is not as large as one might initially suppose. 
The concepts of conservation and extinction defined above relate to the long-run behavior of the resource starting from a given initial stock $y>$ 0 . It is of interest to know in what scenarios the possibility of extinction (conservation) is independent of the initial stock. Thus, it is useful to define the concepts of global extinction (conservation).

Global extinction (conservation) is said to occur if extinction (conservation) occurs from all initial stocks $y>0$. Under global extinction, conservation is impossible no matter how large the current stock of the resource. On the other hand, in a situation of global conservation, the resource is globally safe from extinction.

A weaker form of conservation of interest (compared to global conservation) is one where conservation is guaranteed only if the intial stock exceeds a certain positive stock, which may be called a "safe standard of conservation". A stock $y^{*}>0$ is said to be a safe standard of conservation if:

$$
P\left\{\omega \in \Omega: \lim \inf _{t \geq 0} y_{t}(y, \omega)>0\right\}=1
$$

for all $y>y^{*}{ }^{12}$

In what follows, we establish conditions on the transition function which lead to (i) global extinction, (ii) global conservation; (iii) the existence of a safe standard. We argue later that in our framework, these are the only possible scenarios.

\subsection{Conditions for Extinction and Conservation}

Clearly, if the best transition function lies below the 45-degree line (that is $M(y)<y$ for all $y>0$ ), then we will have global extinction of the resource. In this subsection, our primary interest is in identifying conditions weaker than this which still lead to extinction.

In the stochastic setting of our model, even if the transition function is such that the resource stocks can grow in size under better environmental circumstances, runs of bad shocks can reduce the stocks to levels close to zero infinitely often. One might speculate that the probability with which this occurs depends on the nature of the transition function during "bad" realizations of the shock and the way it compares to the transition during "good"

\footnotetext{
${ }^{12}$ The reason we do not require conservation from the stock $y^{*}$ itself is because we want to allow for a situation where there is a discontinuity in the transition function at $y^{*}$ and the right-hand limit of the function at $y^{*}$ is good enough to guarantee conservation.
} 


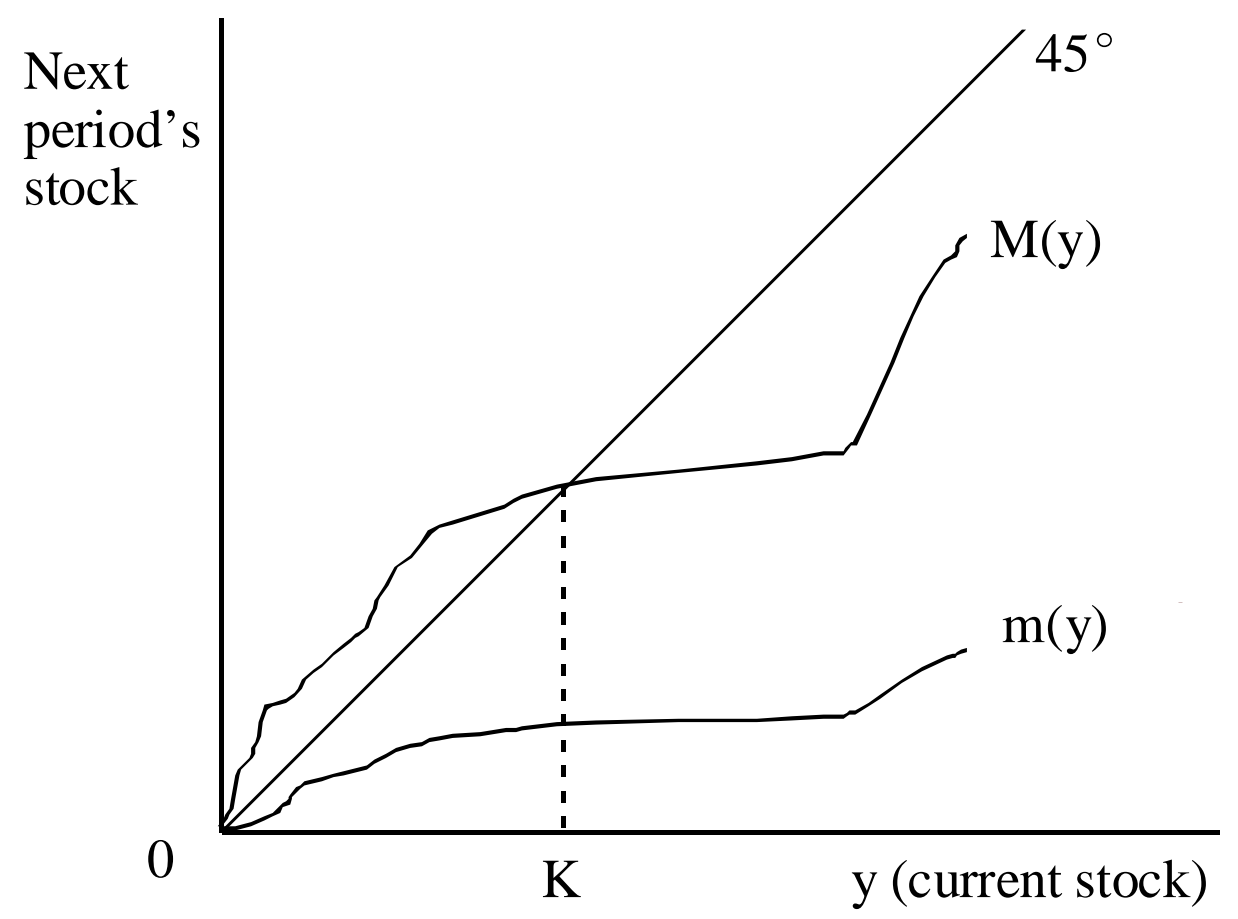

Figure 1: Global Extinction

realizations of the shock as well as the relative weight of these realizations. The surprising result is that the condition for global extinction depends only on the worst transition function $m$ - the behavior of the resource stocks under better environmental conditions is irrelevant.

More specifically, we show that if the function $m$ lies below the 45-degree line at all levels of current stock (Figure 1), global extinction occurs. This reflects the fact that the transition function exhibits "bounded growth" i.e., the optimal stocks are bounded above almost surely by $K$. This means that if one looks at the stochastic process after any particular date, then for any neighborhood of zero, there is a finite run of "bad shocks" which can reduce the resource stock to a level within this neighborhood. Given that the shocks are i.i.d. and that these bad realizations occur with positive probability in any period, it can shown that such a finite run occurs almost surely. This, in 


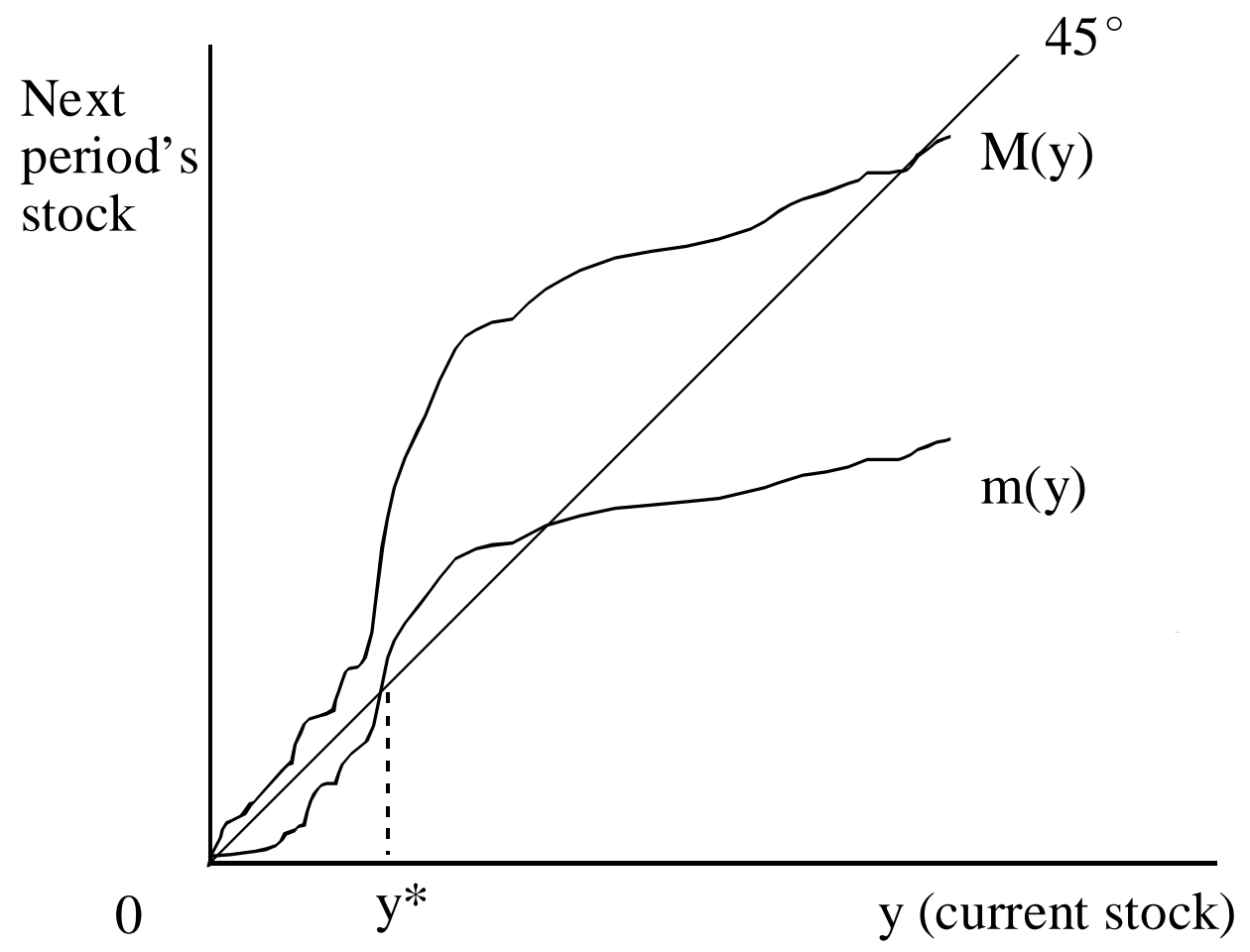

Figure 2: Safe Standard of Conservation

turn, implies that after any finite date, the event that the stocks are bounded away from zero has zero probability.

On the other hand, if $m$ lies on or above the 45-degree line at some positive level of stock $y^{*}$ (Figure 2), then the stochastic process of resource stocks starting from any higher initial stock is almost surely higher than $y^{*}$ and therefore, almost surely bounded away from zero i.e., we have a safe standard of conservation.

Proposition 1 (a) Global extinction occurs if and only if:

$$
\sup \{[m(y) / y]: y \geq h\}<1 \text { for all } h>0
$$


(b) There exists a safe standard of conservation if and only if:

$$
\sup \{[m(y) / y]: y \geq h\} \geq 1 \text { for some } h>0
$$

Further, if (2.9) holds, then there exists $y^{*}>0$ such that $\lim _{y \downarrow y^{*}} m(y) \geq y^{*}$ and for all $y>y^{*}$ and $t \geq 0, y_{t}(y, \omega) \geq y^{*}$ almost surely.

Next, we consider a stronger scenario of global conservation where the stochastic process of stocks is almost surely bounded away from zero no matter how small the initial stock. Given the fact that probability of conservation is non-decreasing in the level of initial stock, it follows that a necessary and sufficient condition for global conservation is that conservation occurs with probability one from stocks close to zero. This, in turn, can be ensured if the transition function is such that for current stocks lying in a neighborhood of zero, the stock size is sustained even under the worst environmental shock i.e., the worst transition function $m(y)$ lies on or above the 45-degree line in a neighborhood of zero (Figure 3).

Proposition 2 Global conservation occurs if there exists $\eta>0$ such that $m(y) \geq y$ for all $y \in(0, \eta)$.

Propositions 1 and 2 show that the worst transition function $m(y)$ is the sole determinant of the nature of conservation/extinction scenarios. Geometrically, the function $\mathrm{m}(\mathrm{y})$ (or precisely, its right hand limit at each $\mathrm{y}$ ) can exhibit one of only three possibilities:

(i) it lies below the 45-degree line at every positive stock as in Figure 1,

(ii) it lies below the 45-degree line at low stocks but eventually crosses the 45-degree line as in Figure 2,

(iii) it lies above the 45-degree line in a neighborhood of zero as in Figure 3.

In case (i), we have global extinction. In case (ii) we have the existence of a safe standard but not necessarily global conservation. In case (iii), global conservation occurs. The three scenarios - global conservation, global extinction and existence of safe standard exhaust the list of possible conservation/extinction scenarios. For example, an outcome where both conservation and extinction occur with positive probability from all positive stocks is impossible.

In case (ii) above, it is difficult to provide any characterization of the probability of conservation from stocks lying below the safe standard. In 


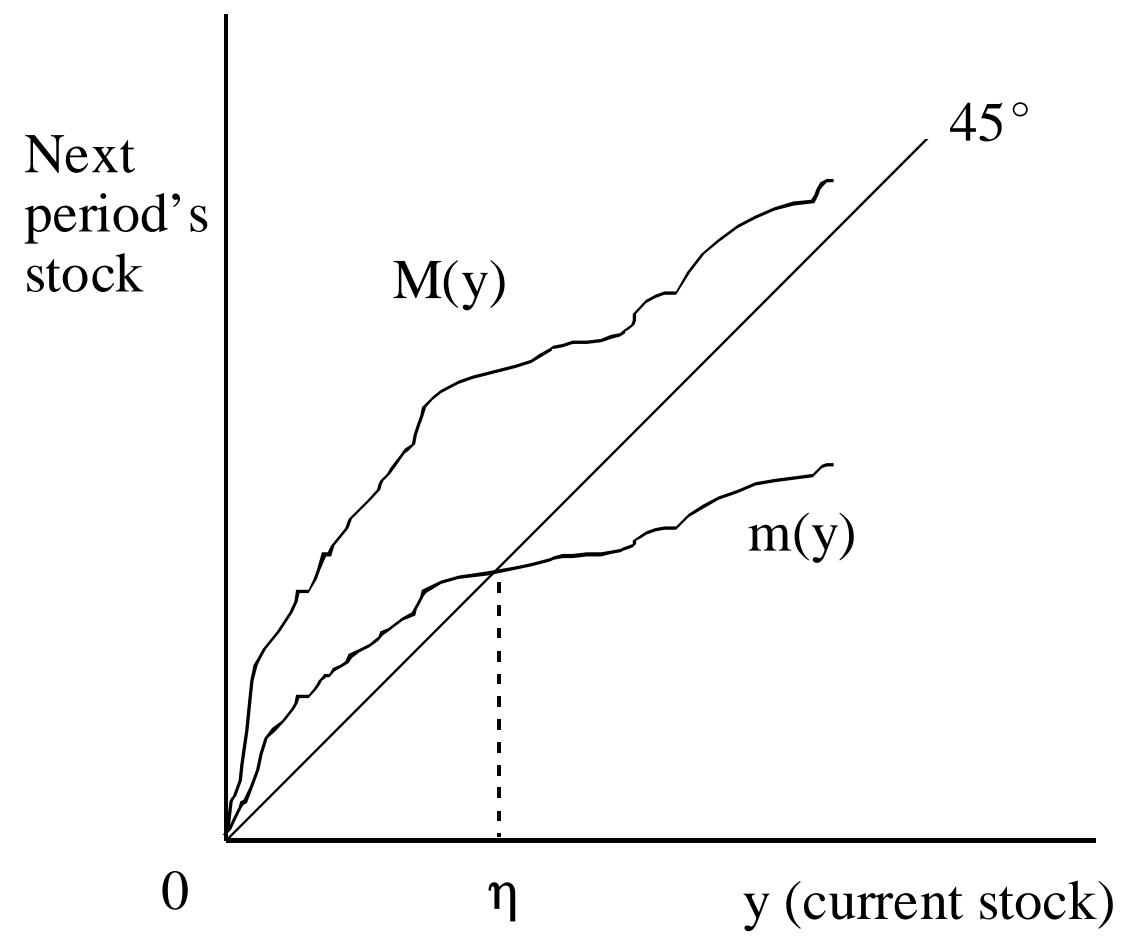

Figure 3: Global Conservation 
particular, it is difficult to rule out the possibility that conservation might occur with probability one from a stock below a safe standard such as $y^{*}$ in Figure 2. This is an important difference between the stochastic and the deterministic versions of the model. In the deterministic framework, we can readily identify the smallest positive stock at which the resource is sustained (where the transition function first crosses the 45-degree line from below) as the minimum safe standard of conservation because extinction must necessarily occur from all lower stocks. This does not necessarily hold in the stochastic model. In the situation depicted in Figure 2, we can only say that $y^{*}$ is a safe standard not necessarily a "minimum" safe standard.

However, there is one special class of transition functions for which the smallest stock at which the resource is sustained under the worst environmental circumstance is in fact, the minimum safe standard of conservation as extinction occurs with positive probability from all smaller stocks. This is the class of transition functions for which, for stocks small enough, the stock size is not sustainable even if the best shock occurs i.e., $M(y)$ lies below the 45-degree line near zero stock. It is well-known that the biological production functions for many species exhibit critical depensation i.e., if the current stock falls below a critical biomass, the stock of the species can only decrease over time even if there is no harvesting. Of course, even if the production function does not exhibit critical depensation, the best transition function may lie below the 45-degree line in a neighborhood of zero if the propensity to consume is high enough in that region.

Figure 4 depicts one such situation. Extinction occurs with probability one from stocks below $\alpha$ as $M(y)<y$ on $(0, \alpha)$. In the figure, $\beta$ is safe standard and is, in fact, the smallest positive stock at which the resource size is sustained with probability one. Observe that for initial stocks lying between $\alpha$ and $\beta$, a finite run of "bad" shocks (close enough to the worst shock) leads the resource stocks to a level below $\alpha$ from where extinction is guaranteed. As this run of "bad shocks" has positive measure, extinction occurs with positive probability from stocks below $\beta$. We can say that $\beta$ is a minimum safe standard of conservation.

For this class of transition functions, if $m(y)<y$ for all $y>0$ (Figure $5)$, then not only does global extinction occur in the sense that the resource stocks come arbitrarily close to zero over time but that, in fact, once they are close enough to zero they decrease in a monotonic fashion to zero i.e., the stocks converge to 0 with probability one. Further, it can be shown that the expected time before the resource falls below any $\varepsilon>0$ is finite. This implies 


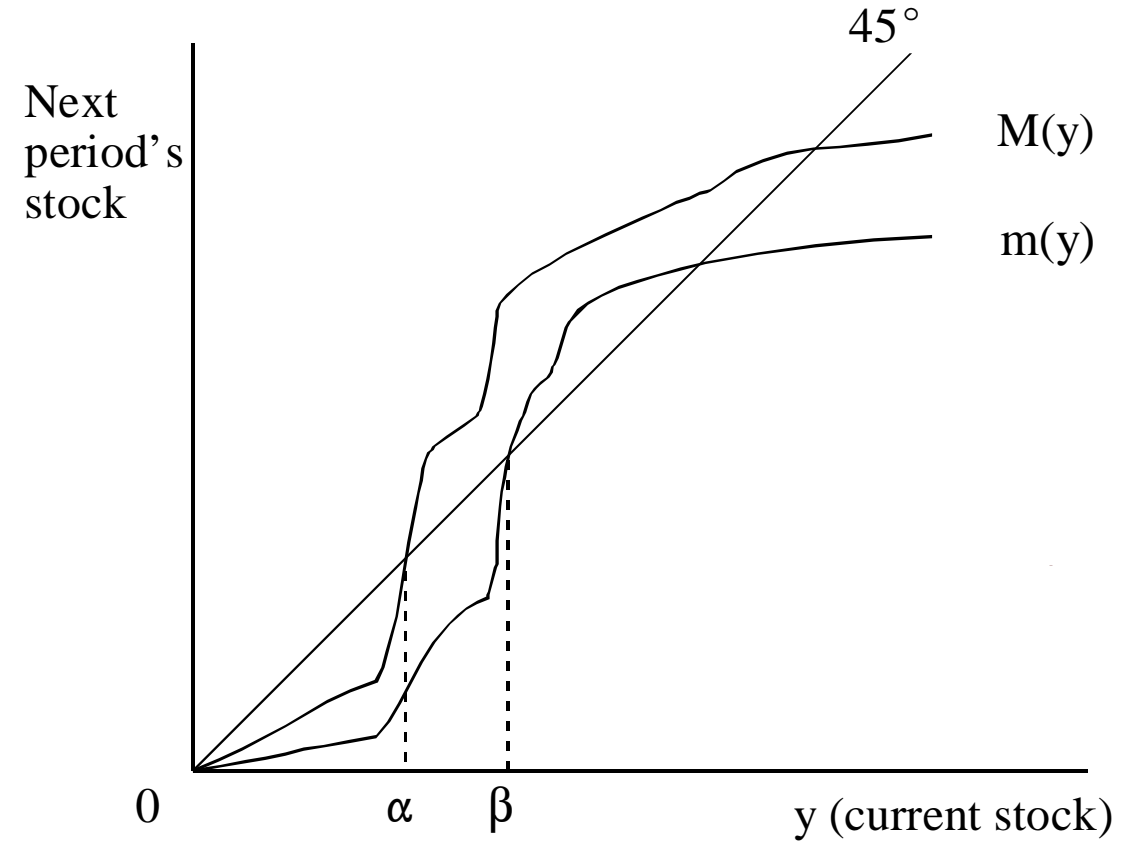

Figure 4: Minimum Safe Standard of Conservation 


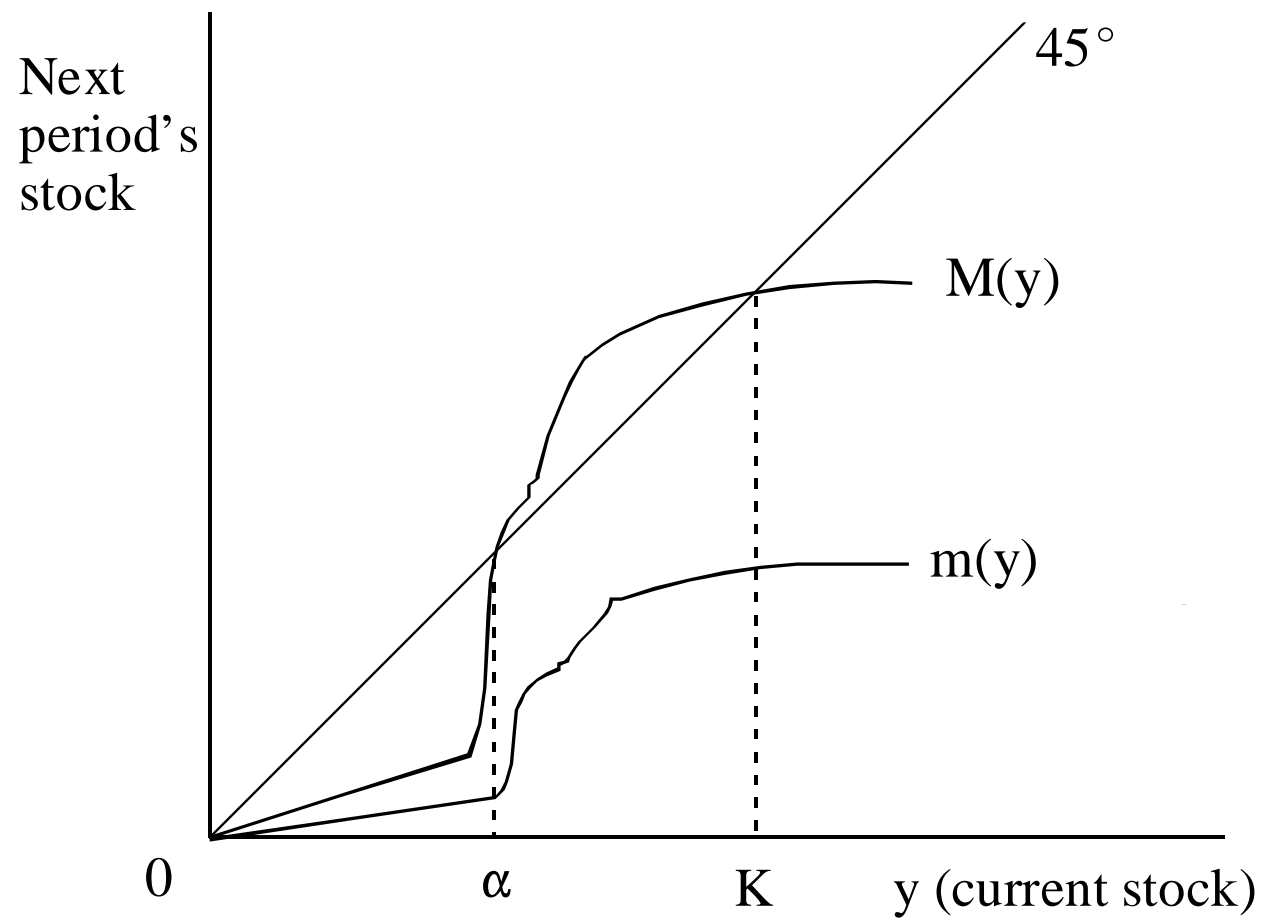

Figure 5: Strong Global Extinction

that, in particular, the stock size reaches the critical neighborhood of zero where $M(y)<y$ in finite expected time and thereafter decreases to zero with probability one. We summarize these results in the following proposition.

Proposition 3 Suppose that there exists $\alpha>0$ such that $M(y)<y$ for all $y \in(0, \alpha)$. Then, the following hold:

(i) If (2.8) holds, then a strong form of global extinction occurs in the sense that the stocks converge to zero almost surely from all initial stocks $y>0$. Further, for any $\varepsilon>0$ and $\tau(y, \omega)=\inf \left\{t \geq 0: y_{t}(y, \omega) \leq \varepsilon\right\}$, $E(\tau(y, \omega))<\infty$ for all $y>0$.

(ii) Suppose (2.9) holds. Let $\beta$ be defined by:

$$
\beta=\inf \left\{\gamma>0: \lim _{y \downarrow \gamma}(m(y) / y) \geq 1\right\}
$$


(a) If $\beta>0$, then $\beta$ is the minimum safe standard of conservation. Extinction occurs with positive probability from stocks below $\beta$ (and with probability one from stocks below $\alpha$ ).

(b) If $\beta=0$, then there is global conservation.

Part (i) of Proposition 3 characterizes a class of transition functions for which stocks converge to zero with probability one. It also ensures two other properties: (i) for almost every sample path, stocks eventually monotonically decrease to zero and (ii) stocks fall below any small positive threshold in finite expected time. We provide below an alternative condition which ensures that stocks converge to zero with probability one, without.ensuring the two additional properties just mentioned. The condition puts a bound on an "average" of the growth rate implied by the transition function.

For every $r \in[a, b]$, let $\lambda(r)=\sup \left\{\frac{G(y, r)}{y}: y>0\right\}$. As long as transition function has a finite slope at zero even under the best environmental circumstance, $\lambda(r)$ is a non-negative real valued function.

Proposition 4 Assume that (i) $\lambda(a)>0$ and $\lambda(b)<+\infty$ and (ii) $E[\ln (\lambda(r))]<$ 0 . Then, resource stocks converge to zero almost surely from all initial stocks $y>0$.

Athreya (2002) establishes a sufficient condition for convergence to zero of a Markov process of the kind used in Proposition 4 above. In our context, his condition reduces to the requirements that (a) for each $r \in I$, $\lim _{y \downarrow 0}[G(y, r) / y]$ exists and is positive and finite; (b) For each $r \in I, \lim _{y \downarrow}[G(y, r) / y]$ $>[G(y, r) / y]$ for all $y>0$, and (c) $E \ln \left[\lim _{y \downarrow 0}\{G(y, r) / y\}\right]<0 .{ }^{13}$ It is easy to check that under these conditions, $\lambda(a)>0$ and $\lambda(b)<+\infty$ and $E[\ln (\lambda(r))]<0$ so that our conditions in Proposition 4 are satisfied. Observe that requirement (b) in Athreya's paper is not satisfied if, for example, $G(y, r)$ is S-shaped in $y$ for each $r$; our conditions do allow for S-shaped transition functions. It is worth noting that the method of proof followed in Athreya (2002) goes through under our weaker conditions.

The results derived in this section are useful in characterizing the possibilities of conservation and extinction in a wide variety of contexts. In the rest of this paper, we shall be primarily concerned with the case where the transition law is determined by the solution to a stochastic dynamic optimization problem by a social planner (or a monopoly exploiter) of the resource.

\footnotetext{
${ }^{13} \mathrm{He}$ also requires continuity of the transition function $G(y, r)$ in $y$.
} 
However, there are many other contexts in which the harvesting rule can be endogenously derived and to which the propositions derived in this section may be applied to obtain fairly tight characterization of the conditions under which extinction or conservation occur in these frameworks. ${ }^{14}$

\section{A Framework for Optimal Resource Man- agement}

In this section, we outline a model where a renewable resource is harvested over time according to the optimal dynamic decisions of a social planner (or monopoly owner). The planner chooses a sequence of resource consumption (or investment) in order to maximize the expected discounted sum of (oneperiod) social welfare over an infinite horizon given a stochastic production function which summarizes the biological growth possibilities and a known distribution of environmental disturbances. The model will be used to answer a broad normative question: under what conditions is it socially efficient to conserve a resource and under what conditions is it optimal to harvest the resource towards extinction? In particular, we characterize the conditions on the preferences and the production function under which the (endogenously determined) optimal decisions are such that the transition law for the resource stocks meets the conditions for extinction and conservation outlined in Propositions 1-3.

\subsection{Production and Welfare Functions}

Time is discrete and is indexed by $t=0,1,2, \ldots \infty$. The initial stock of the resource $y_{0}>0$ is given. At each date $t \geq 0$, the current resource stock $y_{t} \in \mathbb{R}_{+}$is observed and a harvest or consumption level, $c_{t}$, is chosen. The remaining stock represents resource investment or escapement, $x_{t}=$

\footnotetext{
${ }^{14}$ One example is of a framework in which the resource is harvested under open access conditions by price-taking producers, who do not take into account the effect of their current harvest on the future stock, and whose current cost of harvesting depends on the stock size. Given the current stock of the resource in any period, and a period demand function, the static market equilibrium determines the total harvest in each period as a function of the total stock, which, in combination with the biological production function, determines the transition function for the resource stocks. See, for example, Mirman and Spulber (1984).
} 
$y_{t}-c_{t}$. The feasible set for consumption and investment is denoted by $\Gamma(y)=$ $\{(x, c) \mid 0 \leq c, 0 \leq x, c+x \leq y\}$.

There are random environmental shocks to the production (stock-recruitment) process of the renewable resource. We model the random shocks exactly as in Section 2. Let $\left\{r_{t}\right\}$ be an independent and identically distributed random process defined on $(\Omega, \mathcal{F}, P)$, where the marginal distribution is denoted by $\mu$, and where the support of this distribution is given by the interval $[a, b]$ with $0<a<b<\infty$.

The biological growth of the resource is governed by a production function, $f: Y \times I \rightarrow Y$, that determines the resource stock next period (gross output) as a function of current investment in the stock and the environmental shock such that $y_{t+1}=f\left(x_{t}, r_{t+1}\right)$. Resource growth net of investment in the stock is given by $f(x, \rho)-x$. The resource production or growth function is assumed to satisfy the following:

(T.1) For all $r, f(x, r)$ is strictly increasing in $x$; for all $x, f(x, r)$ is nondecreasing in $r$.

(T.2) For all $r, f(0, r)=0$.

(T.3) $f(x, r)$ is continuous in $(x, r)$ on $Y \times I$. For each $r \in[a, b], f(x, r)$ is continuously differentiable in $x$ on $\mathbb{R}_{++}$.

(T.4) There exists $\bar{x}>0$ such that $f(x, b)<x$ for all $x \geq \bar{x}$, and $y_{0} \in$ $(0, \bar{x}]$.

Assumptions T.1-T.3 are standard monotonicity and smoothness restrictions on production. Assumption T.4 is a bounded growth restriction typically associated with a natural carrying capacity for the ecosystem beyond which the resource stock cannot grow.

The lower bound on the intrinsic growth rate (that is, the marginal product at zero investment) is given by the lower right derivative of $f$, which is denoted by $D_{+} f(0, r)=\liminf _{x \downarrow 0} f^{\prime}(x, r)$. Define $\nu=\inf _{r \in I}\left[D_{+} f(0, r)\right]$ to be the lower bound on the intrinsic growth rate over all possible realizations of the random shock. We assume

(T.5) $\nu>0$.

Assumption T.5 ensures that the marginal product is bounded away from zero no matter how small the investment in the resource stock. It allows for cases of critical depensation where the resource production function is not capable of replacing investment from small stocks; that is, $f(x, r)<x$ almost surely for $x$ close to zero in which case extinction is inevitable from small stocks even if the resource is never harvested. Of course, T.5 also encompasses cases where the resource is productive enough to sustain itself in the absence 
of harvesting.

We make no assumption about the concavity of the production function in general. Typically, biological resources are likely to exhibit low "productivity" or growth rate when the biomass is small and it is likely to increase as the biomass expands. That is, the production function is likely to be convex at low levels of investment; as the resource exhibits bounded growth it is reasonable to assume that eventually diminishing returns must set in. For each $r \in[a, b]$, let $S(r)=\{\hat{x} \geq 0:[f(\hat{x}, r) / \hat{x}] \geq[f(x, r) / x]$ for all $x \geq 0\}$,if $\liminf \operatorname{in}_{x \downarrow}[f(x, r) / x]<\infty$; otherwise, let $S(r)=\{0\}$. Define $\hat{x}(r)=\sup \{x: x \in S(r)\}$. Thus, $\hat{x}(r)$ is the highest investment among the set of investments that maximize average productivity corresponding to realization $r$ of the random shock. In the special case of multiplicative shock, $\hat{x}(r)$ is identical for all $r$. We assume that:

(T.6) For each $r \in[a, b], f(x, r)$ is concave in $x$ on $[\hat{x}(r), \infty)$.

Define $\hat{x}=\sup _{r} \hat{x}(r)$. If $\hat{x}>0$, then the production function is nonconcave for at least some $r$. If the production function is concave for all $r$, then $\hat{x}=0$.

Figure 6 illustrates a stochastic, non-concave biological production function that satisfies all the assumptions T.1-T.6.

The existing literature on resource allocation with non-concave production focuses on models where the resource growth function is S-shaped and where the resource can always be sustained from low stocks. The model of resource growth employed here generalizes these two restrictions. First, as illustrated in Figure 6, it allows for the possibility of critical depensation where the resource is incapable of sustaining itself from low stocks. In such cases, the important question is whether economic efficiency implies conservation of the resource from large stocks. Second, the model in this paper considers a broader class of growth functions than those that are S-shaped. Resource growth is allowed to exhibit almost any pattern of increasing and decreasing returns on the interval $[0, \hat{x}(r)]$.

Finally, we impose a technical restriction on the behavior of the production function with respect to changes in realizations of the random shock in a neighborhood of the lowest shock:

(T.7) For any $x>0, \mu\{r: f(x, r)>f(x, a)\}=1$. Further, for any $x^{1}, x^{2}$, with $0<x^{1} \leq x^{2}<\infty$,

$$
\sup _{x \in\left[x^{1}, x^{2}\right]}[f(x, r)-f(x, a)] \rightarrow 0 \text { as } r \downarrow a
$$




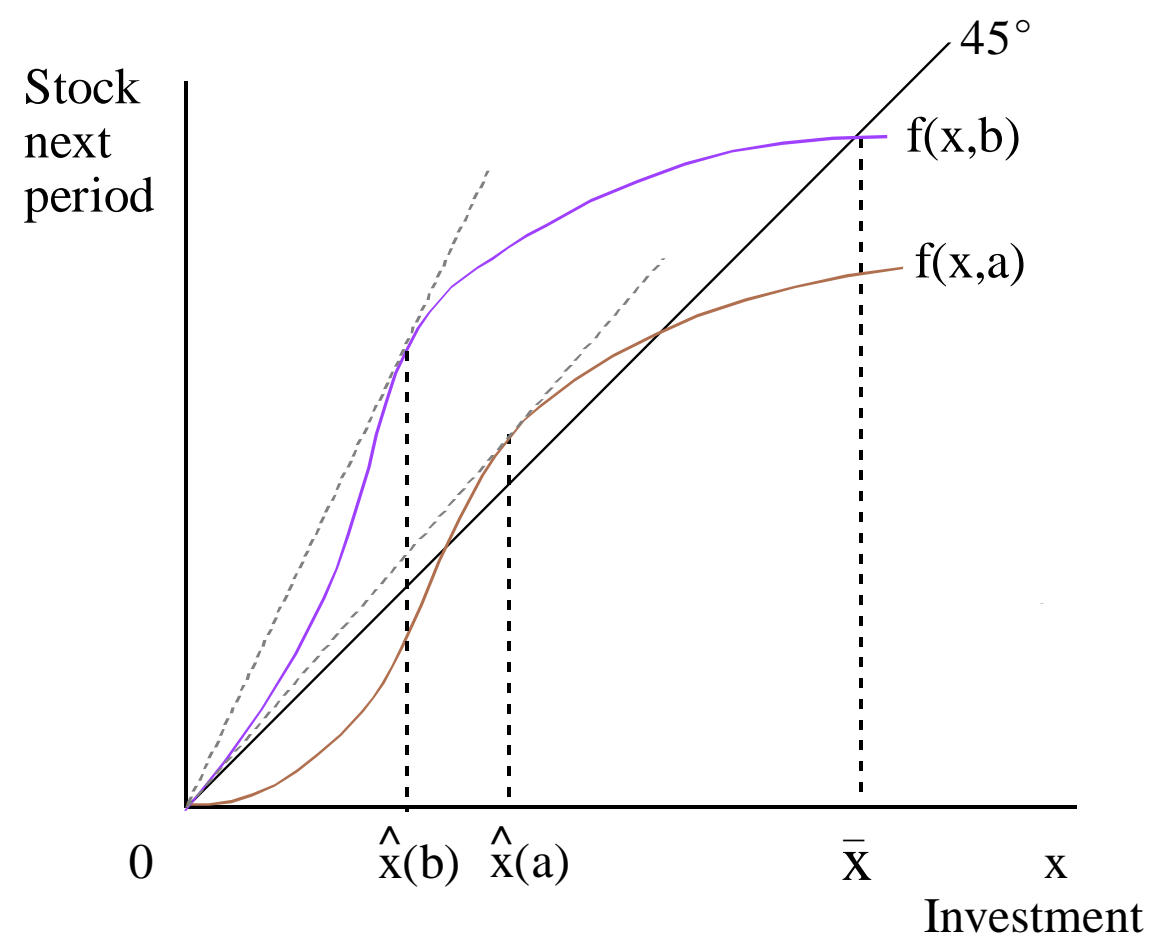

Figure 6: Production Function 
T.7 requires that on any positive interval, the production functions corresponding to various realizations of the random shock converge uniformly to the lowest production function $f(x, a)$. Further, in any period, the probability that next period's stock is exactly equal to that obtainable under the worst production function is zero. This has the effect of putting zero measure on the worst production function (which is stronger than just putting zero measure on the worst realization of the random shock). The assumption is satisfied for the well-known family of production functions which are multiplicatively separable in the investment level and the random shock; that is, are of the form $f(x, r)=F(x) K(r)$, where $F$ is non-decreasing in $x$ and $K$ is continuous in $r$.

The net social welfare in each period depends on current consumption and is denoted by $u(c)$. This welfare function can incorporate consumer and producer surplus from resource harvests. The objective is to maximize the expected discounted sum of social welfare over time, where $\delta \in(0,1)$ is the discount factor. For a monopolist's dynamic optimization problem, $u(c)$ would denote the current profit from from harvest $c$. The welfare function satisfies the following restrictions:

(U.1) $u$ is concave on $Y$.

(U.2) $u$ is twice continuously differentiable on $\mathbb{R}_{++}$.

(U.3) Either there exists a $\xi>0$ such that $u^{\prime}(c)>0$ for all $c \in(0, \xi)$ and $u^{\prime}(c)<0$ for all $c>\xi$, or $u^{\prime}(c)>0$ for all $c \in \mathbb{R}_{++}$.

Assumptions (U.1) and (U.2) are standard. Assumption (U.3) is weaker than the typical assumption in stochastic growth models that $u$ is increasing over the domain of $c$. It implies that welfare is either increasing or unimodal in $c$; that is, there is a unique strictly positive consumption that maximizes $u$. This allows for the possibility that marginal harvest costs might exceed marginal benefits at large harvest levels so that excessive consumption might decrease instantaneous welfare. In case $u^{\prime}(c)>0$ for all $c \in \mathbb{R}_{++}$, we define $\xi=\infty$.

\subsection{Value Function and Optimal Policy}

In general, the decision-maker in the stochastic environment can take decisions dependent on the history of past states and decisions. To formalize this decision-making process, we need to start by defining histories.

The partial history at date $t$ is given by $h_{t}=\left(y_{0}, x_{0}, c_{0}, \ldots, y_{t-1}, x_{t-1}, c_{t-1}, y_{t}\right)$. A policy $\pi$ is a sequence $\left\{\pi_{0}, \pi_{1}, \ldots\right\}$ where $\pi_{t}$ is a conditional probability 
measure such that $\pi_{t}\left(\Gamma\left(y_{t}\right) \mid h_{t}\right)=1$. A policy is Markovian if for each $t, \pi_{t}$ depends only on $y_{t}$. A Markovian policy is stationary if $\pi_{t}$ is independent of $t$.

Associated with a policy $\pi$ and an initial state $y$ is an expected discounted sum of social welfare:

$$
V_{\pi}(y)=E \sum_{t=0}^{\infty} \delta^{t} u\left(c_{t}\right)
$$

where $\left\{c_{t}\right\}$ is generated by $\pi, f$, and $\Psi$ in the obvious manner.

The value function $V(y)$ is defined by:

$$
V(y)=\sup \left\{V_{\pi}(y): \pi \text { is a policy }\right\}
$$

Assumption T.4 ensures that, given any policy $\pi$, we have $V \pi(y)<\infty$ for all $y>0$. We assume that there exists a policy $\pi$ such that $V_{\pi}(y)>-\infty$ for all $\mathrm{y}>0 .{ }^{15}$ Thus, the dynamic optimization problem is well defined and the value is finite from any initial state.

A policy, $\pi^{*}$, is optimal if $V_{\pi^{*}}(y) \geq V_{\pi}(y)$ for all policies $\pi$ and all $y$ and $V_{\pi^{*}}(y)=V(y)$. Standard dynamic programming arguments (see, for example, Strauch [1966]) imply that there exists an optimal solution such that the value function satisfies the functional equation:

$$
V(y)=\sup _{x \in \Gamma(y)}[u(y-x)+\delta E[V(f(x, r)]]
$$

Further, $V$ is increasing and continuous. Let $X(y)$ be the set of maximizers of the expression on the right hand side of (3.1). $X(y)$ is an upperhemicontinuous correspondence that admits a measurable selection. $X(y)$ will be referred to as the (stationary) optimal investment correspondence, while $C(y)=y-X(y)$ will be called the optimal consumption correspondence. The maximum and minimum selections from $X(y)$ are denoted by $X_{m}(y)=\min \{x: x \in X(y)\}$, and $X_{M}(y)=\max \{x: x \in X(y)\}$.

It can be shown that the optimal investment correspondence $X(y)$ has certain monotonicity properties. More precisely, it can be shown that (see, for example, Majumdar, Mitra and Nyarko (1989), Hopenhayn and Prescott [1992, Proposition 2]):

\footnotetext{
${ }^{15}$ If $\nu>1$ or $u(0)>-\infty$, then this always holds. If neither of these conditions hold (a possibility not ruled out by our assumptions), then this can be ensured if the discount factor is smaller than a critical value that depends on $u$ and $\nu$.
} 
Lemma $2 X_{m}(y)$ and $X_{M}(y)$ are non-decreasing in $y$ on $Y$.

In Section 2, the conditions for the occurrence of the alternative conservation/extinction scenarios were ones which were directly imposed on the transition function $G$ and the distribution of the random shocks. When resource stocks are optimally managed over time using an optimal investment function $H(y)$, a measurable selection from $X(y)$, the effective transition function for resource stocks is given by:

$$
y_{t}(y, \omega)=G\left(y_{t-1}(y, \omega), \omega_{t}\right)=f\left(x\left(y_{t-1}(y, \omega)\right), \omega_{t}\right) \text { for } t \geq 1
$$

and $y_{0}(y, \omega)=y$.

Our results in Section 2 were derived under the assumption that $G(y, r)$ is non-decreasing in $y$ and $r$. From (3.2) it is obvious that this requires not only that the production function $f(x, r)$ be non-decreasing in $x$ (which is guaranteed by (T.1)) but also that the optimal investment function $H(y)$ be non-decreasing in current stock $y$. While not every measurable selection from $X(y)$ is monotonic in $y$, Lemma 2 indicates that $X_{m}(y)$ and $X_{M}(y)$ are non-decreasing functions of $y$. Therefore, if we choose either of these two functions as the optimal investment function $x(y)$, then $G(y, r)=f(H(y), r)$ is non-decreasing in $y$. In fact, it is easy to check that:

Proposition 5 For $H(y)=X_{m}(y)$ and for $H(y)=X_{M}(y)$, the optimal transition function $G(y, r)=f(H(y), r)$ satisfies the restrictions (G.1)-(G.4).

Note that the optimal investment correspondence $X(y)$ is upper hemicontinuous but it does not necessarily admit a continuous selection; in particular, both $X_{m}(y)$ and $X_{M}(y)$ may be discontinuous.

In general, the optimal investment and/or consumption need not lie in the interior of the feasible set. The following lemma outlines a condition which guarantees that optimal investment in the resource is strictly positive from all stocks $y>0$.

Lemma 3 Assume that for all $y>0$,

$$
\delta\left\{\liminf _{c \downarrow 0} u^{\prime}(c)\right\} E\left(D_{+} f(0, r)\right)>u^{\prime}(y)
$$

Then $X_{m}(y)>0$ for all $y>0$. 
The condition outlined in this lemma is always satisfied if $u^{\prime}(c) \rightarrow \infty$ as $c \rightarrow 0$. It is also satisfied if the marginal utility of consumption is bounded above but the technology is delta-productive in expected terms; that is, if $E\left(D_{+} f(0, r)\right)>1 / \delta$. The lemma encompasses the standard Inada condition used to guarantee interior investment in classical optimal growth models (see, for example, Brock and Mirman [1972]).

In the classical growth model, the Inada condition also guarantees that optimal consumption is strictly positive, which our condition does not do. In fact, in the stochastic non-convex dynamic optimization framework, guaranteeing that optimal consumption is strictly positive from all initial stocks by a general verifiable restriction on technology and preferences requires us to impose rather strong conditions. If the production function is concave in input, then an assumption such as $u^{\prime}(c) \rightarrow \infty$ as $c \rightarrow 0$ is sufficient to ensure that optimal consumption is strictly positive; this also holds if the production function is non-concave but there is no uncertainty. However, it is not known whether this condition suffices when the technology is both stochastic and non-convex. For example, Majumdar, Mitra and Nyarko (1989) assume that $u(0)=-\infty$ in order to ensure that optimal consumption is always positive.

To conclude this subsection, we note that the stochastic Ramsey-Euler equation holds in case of an interior optimal policy (see Majumdar, Mitra and Nyarko (1989) for a formal proof).

Lemma 4 Let $x_{t}(y, \omega)$ and $c_{t}(y, \omega)$ denote the $t-$ th period investment and consumption respectively under an optimal policy starting from an initial stock $y>0$. If $x_{t}(y, \omega)>0$ and $c_{t}(y, \omega)>0$ a.e. $\omega$, for all $t \geq 0$, then:

$$
u^{\prime}\left(c_{t}(y, \omega)\right)=\delta E\left[u^{\prime}\left(c_{t+1}(y, \omega) f^{\prime}\left(x_{t}(y, \omega), \omega_{t+1}\right) \mid \mathcal{F}_{t}\right] \text { a.e. } \omega .\right.
$$

\section{Extinction and Conservation under Opti- mal Resource Management}

In this section, we outline the conditions on the technology and intertemporal preferences under which the stochastic process of resource stocks generated by the optimal dynamic investment policy is characterized by conservation or extinction. In particular, we examine the conditions which lead to the three alternative scenarios outlined in Section 2, namely, global conservation, global extinction and existence of safe standard of conservation. 


\subsection{Safe Standard of Conservation}

First, we examine the conditions under which there is a safe standard of conservation. For this purpose, we analyze the stochastic process of optimal resource stocks when the optimal investment function selected from $X(y)$ is the lower bound $X_{m}(y)$. For any initial stock $y>0$, consider the sequence of optimal stocks $\left\{y_{t}(y)\right\}$ defined by $y_{0}(y, \omega)=y$ and:

$y_{t}(y, \omega)=G\left(y_{t-1}(y, \omega), \omega_{t}\right)=f\left(X_{m}\left(y_{t-1}, \omega\right), \omega_{t}\right)$ for $t \geq 1$

In terms of the notation in Section 2, the worst transition functions $m(y)$ (corresponding to the lowest environmental shock) is given by:

$$
m(y)=G(y, a)=f\left(X_{m}(y), a\right) \text { for } y \in Y
$$

From Proposition 1, a necessary and sufficient condition for the existence of a safe standard is that $\sup \{(m(y) / y): y \geq h\} \geq 1$ for some $h>0$. In this sub-section, we will establish conditions which ensure that a slightly stronger version of (4.2) holds, namely:

$$
m(y)=f\left(X_{m}(y), a\right) \geq y \text { for some } y>0
$$

Before we go into aspects of the problem that involve intertemporal tradeoffs, there is one class of readily identifiable situations in which conservation is always efficient: if the marginal utility from consumption is negative when investment falls below the level needed to replenish the stock to its current level under the worst productivity shock, then investment by even a myopic agent will be sufficient to at least replenish the stock. Since an optimizing agent never consumes more than a myopic one, this ensures that conservation is efficient.

Proposition 6 Suppose that $u^{\prime}(f(x, a)-x) \leq 0$ for some $x>0$, then the stock $f(x, a)$ is a safe standard of conservation.

Next, we consider situations where the hypothesis of Proposition 6 does not hold; that is, where marginal welfare from consuming an amount which allows the stock to be replenished to its current level even under the worst shock is positive. This is always true if $u$ is a strictly increasing function. To obtain a tight condition for conservation it is necessary to overcome the technical difficulties caused by the non-convexity in the feasible set for the 
dynamic optimization problem when the production function is not concave. Our methodology is to first consider the convexified resource allocation problem obtained by taking the convex hull of the production possibility set for each $r$. Recall the definition of input level $\hat{x}$ in Section $2 ; f(x, r)$ is concave in $x$ for all $x \geq \hat{x}$. We derive a condition that ensures a safe standard of conservation for this modified optimization problem, which lies above $\hat{x}$. This implies that the optimal investments for the modified problem lie in the convex part of the original production possibility set. This allows us to show that the safe standard for the modified problem is also a safe standard for the original problem.

Proposition 7 If there is some $x \geq \hat{x}$ such that $f(x, a)>x$, and:

$$
\inf _{\max (f(x, a)-\xi, 0) \leq z \leq x} \delta E\left[\frac{u^{\prime}(f(x, r)-z)}{u^{\prime}(f(x, a)-z)} f^{\prime}(x, r)\right]>1
$$

then $f(x, a)$ is a safe standard of conservation.

In the deterministic version of this model with an S-shaped production function, the condition for existence of a safe standard of conservation is that at some positive input level, the average productivity of the resource growth function should exceed the discount rate; that is, the production function should be delta-productive (see, among others, Majumdar and Mitra (1982), Dechert and Nishimura (1983)). Note that the welfare function plays no role in this condition for existence of a safe standard. The condition given in Proposition 7 should be looked at as a modification of this deltaproductivity condition in the stochastic model. The requirement is of the form $\delta E\left[\Psi(x, r) f^{\prime}(x, r)\right]>1$ where the term $\Psi(x, r)$ represents welfare effects involving ratio of marginal utilities from consumption and is directly linked to the stochastic nature of the model.

The interpretation of the condition in Proposition 7 is as follows. Given the worst production from some investment level consider a policy that depletes the resource below the original level. If all such policies have a marginal value of consumption strictly less than the expected discounted marginal value of investment, then it must be the case that the optimal investment is one that sustains the stock. Since optimal investment is monotonic in current stock, the stock is conserved under all productivity shocks and from any larger initial stock. The fact that the resource production function is stochastic implies that the marginal value of investment is evaluated over 
all possible realizations of the environmental disturbance. The ratio of the marginal value of investment to the marginal utility of current consumption generally differs across states of nature. The welfare effects associated with $\Psi(x, r)$ represent a lower bound on the ratio of the marginal gain in value from an increase in investment to the marginal welfare sacrificed by the corresponding reduction in current consumption. Thus, unlike the deterministic case, the welfare function plays a crucial role in determining whether or not a safe standard exists in the stochastic model.

Note that $\Psi(x, r)<1$ for each $r>a$ so that the condition is actually stronger than requiring that delta-productivity hold in "expected terms". This is not surprising once we consider the fact that the condition is designed to ensure that even under the worst environmental shock, the stock size is sustained from a certain level onwards. ${ }^{16}$ Also observe that $\Psi(x, r)=1$ if there is no production uncertainty and in that case the condition in Proposition 7 simply reduces to the standard delta-productivity condition found in deterministic models.

\subsection{Global Conservation}

Now, we examine the conditions under which the resource is conserved from any initial stock and along any realized path of the random shock. For this purpose we continue to focus on the optimal policy generated by $X_{m}(y)$ and that (4.1) and (4.2) hold. From Proposition 2, we know that global conservation can be ensured if $m(y) \geq y$ for all $y$ in some neighborhood of zero. This requires in particular that $f(x, a) \geq x$ in a neighborhood of zero: the resource production function should not be characterized by critical depensation.

In our analysis of a safe standard of conservation, we were able to obtain considerable leverage by taking the convex hull of production possibilities and by studying the modified dynamic optimization problem on a convex feasible set. This was a fruitful approach because the best hope for finding a safe standard of conservation is in the region where average productivity of the

\footnotetext{
${ }^{16}$ It is not very surprising that in environments where the resource growth is stochastic, it is not possible to ensure the efficiency of conservation by focusing solely on the productivity of the resource relative to the discount rate. In a classical stochastic optimal growth model, Mirman and Zilcha (1976) develop an example where the production function has infinite marginal product at zero for all realizations of the random shock and yet it is possible that $m(y)<y$ for all $y>0$, and there is no safe standard of conservation.
} 
resource is maximized. Further, for stocks above this region, and for the class of resource production functions admissible under T.1-T.7, the convex hull coincides with the original production possibilities for the resource. Unfortunately, this approach is not useful in analyzing global conservation, because it requires conservation in a neighborhood of zero, which is precisely where resource production possibilities are most likely to exhibit non-convexities.

Observe that strictly positive investment is a necessary requirement for global conservation. The condition given in Lemma 3 in Section 3 is sufficient for this to occur. Optimal investment may, however, be positive under weaker conditions and so we shall directly make that assumption.

Proposition 8 Suppose that $\nu>1$, and that optimal investment $X_{m}(y)>$ 0 from all initial stock $y>0$. Then global conservation is optimal if the following condition holds:

$$
\lim \inf _{x \downarrow 0} \delta E\left[\frac{u^{\prime}(f(x, r)}{u^{\prime}(f(x, a)-x)} f^{\prime}(x, r)\right]>1
$$

Proposition 8 is the natural analogue of Proposition 7 for stocks approaching zero. The basic idea underlying the condition in Proposition 8 is straightforward: if under the worst production shock from stocks close to zero, a policy that further depletes the stock has a marginal value of consumption that is strictly less than the (expected discounted) marginal value of zero investment, then optimal investment must be one that leads to conservation.

In deterministic versions of the model, the condition for global conservation is typically a requirement that the marginal productivity at zero (the intrinsic growth rate) exceed the discount rate. One can look at the expression:

$$
\liminf \inf _{x \downarrow \mathbf{0}} \delta E\left[\frac{u^{\prime}(f(x, r)}{u^{\prime}(f(x, a)-x)} f^{\prime}(x, r)\right]
$$

as the expected welfare-modified intrinsic growth rate of the specie which has to exceed the discount rate in order for global conservation to be optimal. Note that the expression above is smaller than the expected marginal productivity at zero. Therefore, as in the case of the condition for safe standard, our condition for global conservation is stronger than "expected deltaproductivity". Finally, note that unlike the condition outlined for existence of safe standard, for the case where there is no uncertainty, the condition for global conservation does not reduce to the corresponding condition in the deterministic version of the model. 


\subsection{Global Extinction}

In this final subsection, we outline the conditions under which it is optimal to lead the resource towards extinction from all stocks with probability one. For this purpose we shall focus on the optimal investment function given by $X_{M}(y)$, so that our condition would ensure global extinction for any measurable selection from $X(y)$. From Proposition 1, we know that global extinction occurs if and only if the worst transition function $m(y)$ is such that $\sup \{(m(y) / y): y \geq h\}<1$ for all $h>0$. The worst transition function will satisfy this property for any optimal policy selection from $X(y)$ if :

$$
\sup \left\{\left(f\left(X_{M}(y), a\right) / y\right): y \geq h\right\}<1 \text { for all } h>0
$$

In the deterministic version of the model, global extinction occurs if the marginal productivity of investment never exceeds $(1 / \delta)$ and in such case, optimal stocks always converge to zero. In our stochastic version, we will show that global extinction is optimal whenever the resource is not deltaproductive from any stock in an expected sense. The proof of this result uses the Ramsey-Euler equation and requires that optimal consumption be positive. Also observe that if for any $x>0$, marginal utility of consuming an amount $[f(x, a)-x]$ is negative, then as noted in Proposition 6, there would always be a safe standard of conservation (no matter how severe the discounting). Therefore, our condition for global extinction will also require that $f(x, a)-x<\xi$ for all relevant $x$.

Proposition 9 Suppose that optimal consumption is strictly positive from all initial stocks below $\bar{x}$ (that is, $X_{M}(y)<y$ for all $\left.y \in(0, \bar{x}]\right)$. Suppose, in addition, that $f(x, a)-x<\xi$ for all $x \in(0, \bar{x}]$. Then, global extinction occurs along any optimal policy if $\delta E\left[f^{\prime}(x, r)\right]<1$ for all $x \in(0, \bar{x}]$.

In Propositions 7 and 8 we have seen that the sufficient conditions for conservation in the stochastic model are stronger than the "expected" version of the conditions for conservation in comparable deterministic models. Thus, simply requiring the technology to be delta-productive in expected terms is not sufficient. It is therefore intuitive that the conditions for extinction to be optimal ought to be weaker than the "expected" version of the conditions found in deterministic model. Thus, if the resource is never delta-productive in expected terms (that is, the expected growth rate of the specie is always lower than the discount rate), then this ought to be sufficient for almost sure extinction to be efficient from all initial stocks. 
Finally, consider the special case where the production function exhibits critical depensation; that is, $f(x, b)<x$ for $x$ small enough. This implies that from current stock levels close enough to zero, it is optimal to decrease the stocks to zero almost surely. For such a production function, if the hypothesis of Proposition 9 holds then we have (directly from Proposition 3) that not only does global extinction occur but optimal stocks always converge to zero with probability one. Further, optimal stocks fall below any $\varepsilon>0$ in finite expected time.

\section{Proofs}

\section{Proof of Lemma 1:}

We are given that:

$$
d=\sup \left\{G(y, a) / y: y \in\left[p, p^{\prime}\right]\right\}<1
$$

Choose $\zeta>0$ and small enough so that $(\zeta / p)<(1-d)$. Given (G.4), there is $\lambda \in(0,(b-a))$ such that:

$$
\sup \left\{G(y, a+\lambda)-G(y, a): y \in\left[p, p^{\prime}\right]\right\}<\zeta
$$

so that for all $y \in\left[p, p^{\prime}\right]$,

$$
\{G(y, a+\lambda)-G(y, a)\} / y<(\zeta / p)<(1-d)
$$

Thus, we we have for all $y \in\left[p, p^{\prime}\right]$,

$$
\begin{aligned}
G(y, a+\lambda) / y & =[G(y, a+\lambda)-G(y, a)] / y+[G(y, a) / y] \\
& <(\zeta / s)+d
\end{aligned}
$$

Using (5.1) and (5.2), we get:

$$
\sup \left\{[G(y, a+\lambda) / y]: y \in\left[p, p^{\prime}\right]\right\} \leq(\zeta / s)+d<1
$$

Now, choosing $\theta \in\left(\sup \left\{[G(y, a+\lambda) / y]: y \in\left[p, p^{\prime}\right]\right\}, 1\right)$, the lemma is proved. Proof of Proposition 1:

(a) Suppose that $\sup \{(m(y) / y): y \geq h\}=\sup \{G(y, a) / y: y \geq h\}<1$ for all $h>0$. We want to show that:

$$
P\left\{\omega \in \Omega: \liminf _{t \geq 0} y_{t}(y, \omega)>0\right\}=0 \text { for all } y>0
$$


Under (G.2), it is sufficient to show this for all $y \in(0, K]$. Suppose that there is some $y \in(0, K]$, such that:

$$
P\left\{\omega \in \Omega: \lim \inf _{t \geq 0} y_{t}(y, \omega)>0\right\}>0
$$

Then, there is some $s>0$ such that:

$$
P\left\{\omega \in \Omega: \lim \inf _{t \geq 0} y_{t}(y, \omega)>s\right\}>0
$$

Observe that:

$$
\begin{aligned}
& \left\{\omega \in \Omega: \liminf _{t \geq 0} y_{t}(y, \omega)>s\right\} \\
& =\{\omega \in \Omega: \text { there exists } T(\omega)<\infty \text { such that } \\
& \left.y_{t}(y, \omega)>s \text { for all } t>T(\omega)\right\} \\
& \subset \cup_{T=0}^{\infty}\left\{\omega \in \Omega: y_{t}(y, \omega)>s \text { for all } t>T\right\}
\end{aligned}
$$

Thus, we have:

$$
\left.\begin{array}{l}
P\left\{\omega \in \Omega: \liminf _{t \geq 0} y_{t}(y, \omega)>s\right\} \\
\leq \sum_{t=0}^{\infty} P\left\{\omega \in \Omega: y_{t}(y, \omega)>s \text { for all } t>T\right\}
\end{array}\right\}
$$

We now need to estimate the probabilities appearing in the sum in the inequality (5.7). By Lemma 1 , there are $\lambda \in(0,(b-a))$ and $\theta \in(0,1)$, such that:

$$
G(y, r)<\theta y \text { for all } r \in[a, a+\lambda] \text { and all } y \in[s, K]
$$

Let $N$ be the smallest positive integer such that $\theta^{N} K<s$. Let $A$ be the event defined by:

$$
A=\left\{\omega \in \Omega: \exists t \geq T \text { for which } \omega_{t+i} \in[a, a+\lambda] \text { for } i=1, \ldots, N\right\}
$$

Observe that on the set $A$, we have $y_{t+N}(K, \omega)<s$ for some $t \geq T$. Since $y_{t}(y, \omega) \leq K$ for almost every $\omega \in \Omega$ for all $t \geq T$, we obtain:

$$
\begin{aligned}
P\{\omega & \left.\in \Omega: \exists t \geq T \text { for which } y_{t}(y, \omega) \leq s\right\} \\
& \geq P\left\{\omega \in \Omega: \exists t \geq T \text { for which } \omega_{t+i} \in[a, a+\lambda] \text { for } i=1, \ldots, N\right\} \\
& =1
\end{aligned}
$$

the last equality in (5.9) following from the fact that $\left\{r_{t}\right\}$ is a sequence of iid random variables with marginal distribution $\mu$, and $\mu\{r \in[a, a+\lambda]\}>0$. Thus, we have:

$$
P\left\{\omega \in \Omega: y_{t}(y, \omega)>s \text { for all } t \geq T\right\}=0 \text { for each } T \geq 0
$$


which implies that the right-hand side of (5.7) is zero. This contradicts (5.6) and hence (5.5), concluding the proof.

(b) By (a), if there is a safe standard of conservation, then:

$$
\sup \{(m(y) / y): y \geq h\} \geq 1 \text { for some } h>0
$$

To establish the converse, suppose there is some $h>0$ such that:

$$
\sup \{(G(y, a) / y): y \in[h, K]\} \geq 1
$$

We will show that in this case there exists a safe standard of conservation. Since $\mathrm{G}$ is non-decreasing in $y$, it is easy to check that there are two possibilities: (i) there exists $y^{*} \geq h$ such that $G\left(y^{*}, a\right) \geq y^{*}$ and that for all $y>y^{*}$, $G(y, a) \geq y^{*}$; (ii) $G(y, a)<y$ for all $y \geq h$, but there exists $y^{*} \geq h$ such that $\lim _{y \downarrow y^{*}} G(y, a)=y^{*}$, and for all $y>y^{*}, G(y, a) \geq y^{*}$.

In case (i), consider any $y \geq y^{*}$. We claim that for all $t \geq 0, y_{t}(y, \omega)>y^{*}$ for almost every $\omega \in \Omega$. Clearly, this is true for $t=0$. We suppose this is true for $t=0, \ldots, T$. Then, we have $y_{T+1}(y, \omega)=G\left(y_{T}(y, \omega), \omega_{T+1}\right) \geq G\left(y^{*}, a\right) \geq$ $y^{*}$ for almost every $\omega \in \Omega$. This proves our claim by induction, and shows that $y^{*}$ is a safe standard of conservation.

In case (ii), consider any $y>y^{*}$. We claim that for all $t \geq 0, y_{t}(y, \omega)>y^{*}$ for almost every $\omega \in \Omega$. Clearly, this is true for $t=0$. We suppose this is true for $t=0, \ldots, T$. Then, using (G.4) we have $y_{T+1}(y, \omega)=G\left(y_{T}(y, \omega), \omega_{T+1}\right)>$ $G\left(y_{T}(y, \omega), a\right)$ for almost every $\omega \in \Omega$. Since $y_{T}(y, \omega)>y^{*}$ by the induction hypothesis, $G\left(y_{T}, a\right) \geq \lim _{y \downarrow y^{*}} G(y, a)=y^{*}$ (since $G$ is non-decreasing in $y$ ) . Thus, we have $y_{T+1}(y, \omega)>y^{*}$ for almost every $\omega \in \Omega$. This proves our claim by induction, and shows that $y^{*}$ is a safe standard of conservation.

\section{Proof of Proposition 2:}

Since the function $m$ is non-decreasing, we have $m(y)>0$ for all $y>0$. This means that for all $y>0$, we have $y_{t}(y, \omega)>0$ for almost every $\omega \in \Omega$.

Given any initial stock $y \geq \eta$, we claim that for all $t \geq 0, y_{t}(y, \omega) \geq \eta$ for almost every $\omega \in \Omega$. Clearly, this is true for $t=0$. We suppose this is true for $t=0, \ldots, T$. Then, since $G$ is non-decreasing in $y$, we have $y_{T+1}(y, \omega)=$ $G\left(y_{T}(y, \omega), \omega_{T+1}\right) \geq G(\eta, a) \geq \eta$ for almost every $\omega \in \Omega$. This proves our claim by induction.

Given any initial stock $y \in(0, \eta)$, we claim that for all $t \geq 0, y_{t}(y, \omega) \geq y$ for almost every $\omega \in \Omega$. Clearly, this is true for $t=0$. We suppose this is true for $t=0, \ldots, T$. Then, since $G$ is non-decreasing in $y$, we have $y_{T+1}(y, \omega)=$ 
$G\left(y_{T}(y, \omega), \omega_{T+1}\right) \geq G(y, a) \geq y$ for almost every $\omega \in \Omega$. This proves our claim by induction.

Using the above two claims, we see that, given any initial stock $y>0$, we have $y_{t}(y, \omega) \geq \min \{y, \eta\}$ for almost every $\omega \in \Omega$.

\section{Proof of Proposition 3:}

(i) Choose $\varepsilon$ in $(0, \alpha)$. It is sufficient to show the result for $y \in(\varepsilon, K]$, where $K$ is defined in (2.1). Pick any $\bar{y} \in(\varepsilon, K]$. Since (2.8) holds, using Proposition 1, we know that global extinction occurs, and hence, we have:

$$
P\left\{\omega \in \Omega: \exists t \text { for which } y_{t}(\bar{y}, \omega) \leq \varepsilon\right\}=1
$$

Also, if $y_{t}(\bar{y}, \omega) \leq \varepsilon$, then $y_{t+1}(\bar{y}, \omega)=G\left(y_{t}(\bar{y}, \omega), \omega_{t+1}\right) \leq G\left(y_{t}(\bar{y}, \omega), b\right)=$ $M\left(y_{t}(\bar{y}, \omega)\right)<y_{t}(\bar{y}, \omega)$, and so $\left\{y_{t}(\bar{y}, \omega)\right\}$ must converge. Thus, we have:

$$
P\left\{\omega \in \Omega: \lim _{t \rightarrow \infty} y_{t}(\bar{y}, \omega)=0\right\}=P\left\{\omega \in \Omega: \lim \inf _{t \rightarrow \infty} y_{t}(\bar{y}, \omega)=0\right\}=1
$$

We now show that the expected time to extinction is finite. Since $(2.8)$ holds, we can use Lemma 1 to obtain $\lambda \in(0,(b-a))$ and $\theta \in(0,1)$, such that:

$$
G(y, r)<\theta y \text { for all } r \in[a, a+\lambda] \text { and all } y \in[\varepsilon, K]
$$

Let $N$ be the smallest positive integer such that $\theta^{N} K<\varepsilon$. For each $t \geq 0$, let $B_{t}$ be the event defined by:

$$
B_{t}=\left\{\omega \in \Omega: \omega_{t+i} \in[a, a+\lambda] \text { for } i=1, \ldots, N\right\}
$$

Observe that for each $t \geq 0$, for $\omega \in B_{t}$, we have $y_{t+N}(y, \omega) \leq y_{t+N}(K, \omega)<\varepsilon$. Let $q=\mu\{r \in[a, a+\lambda]\}$; then, $0<q<1$.

Now, for any $t \geq 0$, we have:

$$
\begin{aligned}
P\{\omega & \left.\in \Omega: y_{t+N}(y, \omega)<\varepsilon\right\} \geq \\
P\{\omega & \left.\in \Omega: y_{t}(y, \omega)<\varepsilon\right\}+P\left\{\omega \in \Omega: y_{t}(y, \omega) \geq \varepsilon\right\} P\left(B_{t}\right) \\
& =P\left\{\omega \in \Omega: y_{t}(y, \omega)<\varepsilon\right\}+P\left\{\omega \in \Omega: y_{t}(y, \omega) \geq \varepsilon\right\} q^{N}
\end{aligned}
$$

so that:

$$
P\left\{\omega \in \Omega: y_{t+N}(y, \omega)<\varepsilon\right\} \leq P\left\{\omega \in \Omega: y_{t}(y, \omega) \geq \varepsilon\right\}\left(1-q^{N}\right)
$$

Define for $t \geq 0, A_{t}=\left\{\omega \in \Omega: y_{t}(y, \omega) \geq \varepsilon\right\}$. Denote $\left(1-q^{N}\right)$ by $v$; so $v \in(0,1)$. Then, for any $k \geq 0$ and $j \geq 1$, (5.11) implies that:

$$
P\left(A_{N(k+1)+j}\right) / P\left(A_{N k+j}\right) \leq v
$$


Since $\tau(y, \omega)$ is a positive integer valued random variable, we have:

$$
E(\tau(y, \omega))=\sum_{t=1}^{\infty} P\left(A_{t}\right)
$$

by using the corollary in Chung $(1974$, p.43). For $j=1, \ldots, N$, define:

$$
C_{j}=\sum_{k=0}^{\infty} P\left(A_{j+k N}\right)
$$

It follows from (5.12) that $C_{j}<\infty$ for each $j \in\{1, \ldots, N\}$. Using (5.13) and (5.14), we get:

$$
E(\tau(y, \omega))=\sum_{t=1}^{\infty} P\left(A_{t}\right)=\sum_{j=1}^{N} C_{j}<\infty
$$

which completes our proof.

(ii) (a) When $\beta>0$, for any $\gamma \in(0, \beta)$, we have:

$$
\lim _{y \downarrow \gamma}(m(y) / y)<1
$$

Pick any $\alpha^{\prime} \in(0, \beta)$ and any $\gamma \in\left(\alpha^{\prime}, \beta\right)$. We claim that:

$$
\sup \left\{(m(z) / z): z \in\left[\alpha^{\prime}, \gamma\right]\right\}<1
$$

Suppose on the contrary there is a sequence $z^{s} \in\left[\alpha^{\prime}, \gamma\right]$ such that $\left[m\left(z^{s}\right) / z^{s}\right] \geq$ $1-(1 / s)$ for $s \in \mathbb{N}$. Since $\left[\alpha^{\prime}, \gamma\right]$ is compact, there is a convergent subsequence of it which converges to some $\bar{z} \in\left[\alpha^{\prime}, \gamma\right]$. Without loss of generality, we can suppose that $z^{s} \rightarrow \bar{z}$ as $s \rightarrow \infty$. Defining $y^{s}=\max \left(z^{s}, \bar{z}\right)$, we have $y^{s} \downarrow \bar{z}$, and:

$$
\lim _{y^{s} \downarrow \bar{z}}\left(m\left(y^{s}\right) / y^{s}\right) \geq 1
$$

which contradicts (5.15), and establishes (5.16).

Pick any $y^{\prime} \in(0, \alpha)$. Since $M(z)<z$ for $z \in(0, \alpha)$, we have $y_{s+1}\left(y^{\prime}, \omega\right)<$ $y_{s}$ for $s \geq 0$, for every $\omega \in \Omega$, and $y_{t}\left(y^{\prime}, \omega\right)$ converges to some $\bar{y}(\omega) \in[0, \alpha)$ as $t \rightarrow \infty$. We claim now that:

$$
P\left\{\omega \in \Omega: \lim _{t \rightarrow \infty} y_{t}\left(y^{\prime}, \omega\right)>0\right\}=0
$$

Suppose, on the contrary that:

$$
P\left\{\omega \in \Omega: \lim _{t \rightarrow \infty} y_{t}\left(y^{\prime}, \omega\right)>0\right\}>0
$$


Then, there is $N \in \mathbb{N}$, with $N>(1 / \alpha)$, such that:

$$
P\left\{\omega \in \Omega: \lim _{t \rightarrow \infty} y_{t}\left(y^{\prime}, \omega\right)>(1 / N)\right\}>0
$$

Denote $\left\{\omega \in \Omega: \lim _{t \rightarrow \infty} y_{t}\left(y^{\prime}, \omega\right)>(1 / N)\right\}$ by $D$.

Since $(1 / N)<\alpha$, we have from $(5.16)$ :

$$
\sup \{(m(z) / z): z \in[(1 / N), \alpha]\}<1
$$

So, we can use Lemma 1 to obtain $0<\lambda^{\prime}<(b-a)$ and $\theta^{\prime} \in(0,1)$, such that:

$$
G(z, r)<\theta^{\prime} z \text { for all } r \in\left[a, a+\lambda^{\prime}\right] \text { and all } z \in[1 / N, \alpha]
$$

Define $q^{\prime}=\mu\left\{r \in\left[a, a+\lambda^{\prime}\right]\right\}$; then $q^{\prime} \in(0,1)$.

For each $n \in \mathbb{N}$, define:

$$
B_{n}=\left\{\omega \in B: \omega_{s} \in\left(a+\lambda^{\prime}, b\right] \text { for } s \geq n\right\}
$$

and :

$$
B=\cup_{n=1}^{\infty} B_{n}
$$

Clearly, $\mu\left\{r \in\left(a+\lambda^{\prime}, b\right]\right\}=\left(1-q^{\prime}\right) \in(0,1)$, and so $P\left(B_{n}\right)=0$ for each $n \in \mathbb{N}$. Consequently, we have:

$$
P(B) \leq \sum_{n=1}^{\infty} P\left(B_{n}\right)=0
$$

Define $\varepsilon=\left[\left(1 / \theta^{\prime}\right)-1\right](1 / N)$. For each $\omega \in D$, there is $T(\omega)$, such that for all $t \geq T(\omega)$, we have:

$$
0 \leq\left[y_{t}\left(y^{\prime}, \omega\right)-\bar{y}(\omega)\right] \leq \varepsilon
$$

Then, for $t \geq T(\omega)$, we get:

$$
\begin{aligned}
\bar{y}(\omega) & \leq y_{t+1}\left(y^{\prime}, \omega\right)=\left[\frac{G\left(y_{t}\left(y^{\prime}, \omega\right), \omega_{t+1}\right)}{y_{t}\left(y^{\prime}, \omega\right)}\right] y_{t}\left(y^{\prime}, \omega\right) \\
& \leq\left[\frac{G\left(y_{t}\left(y^{\prime}, \omega\right), \omega_{t+1}\right)}{y_{t}\left(y^{\prime}, \omega\right)}\right][\bar{y}(\omega)+\varepsilon]
\end{aligned}
$$

Thus, we have for $t \geq T(\omega)$,

$$
\left[\frac{G\left(y_{t}\left(y^{\prime}, \omega\right), \omega_{t+1}\right)}{y_{t}\left(y^{\prime}, \omega\right)}\right] \geq \frac{\bar{y}(\omega)}{[\bar{y}(\omega)+\varepsilon]} \geq \frac{(1 / N)}{[(1 / N)+\varepsilon]}=\theta^{\prime}
$$


This implies, using (5.19), that for all $t \geq T(\omega), \omega_{t+1} \in\left(a+\lambda^{\prime}, b\right]$. That is, if $\omega \in D$, then $\omega \in B_{n}$ for some $n \in \mathbb{N}$, and so $\omega \in B$. But, since $P(B)=0$, we must have $P(D)=0$, which contradicts (5.18) and establishes (5.17). That is, we have:

$$
P\left\{\omega \in \Omega: \lim _{t \rightarrow \infty} y_{t}\left(y^{\prime}, \omega\right)=0\right\}=1
$$

Pick any $y \in[\alpha, \beta)$. Given (5.16), we have in particular:

$$
\sup \{(m(z) / z): z \in[\alpha, y]\}<1
$$

Thus, we can again use Lemma 1 to obtain $\lambda \in(0,(b-a))$ and $\theta \in(0,1)$ such that:

$$
G(z, r)<\theta z \text { for all } r \in[a, a+\lambda] \text { and all } z \in[\alpha, y]
$$

Let $N$ be the smallest positive integer such that $\theta^{N} \beta<\alpha$. Let $B$ be the event defined by:

$$
B=\left\{\omega \in \Omega: \omega_{i} \in[a, a+\lambda] \text { for } i=1, \ldots, N\right\}
$$

Observe that for $\omega \in B$, we have $y_{N}(y, \omega) \leq \theta^{N} \beta<\alpha$. Let $q=\mu\{r \in$ $[a, a+\lambda]\}$; then, we have $0<q<1$, and $P(B)=q^{N}$. Thus, $y_{N}(y, \omega)<\alpha$ with probability at least $q^{N}>0$. Thus, by (5.20), we have:

$$
P\left\{\omega \in \Omega: \lim _{t \rightarrow \infty} y_{t}(y, \omega)=0\right\} \geq P(B)=q^{N}>0
$$

(ii)(b) If $\beta=0$, then there is a sequence $\left\{\gamma^{s}\right\}$, with $\gamma^{s}>0$ for all $s \in \mathbb{N}$, and $\gamma^{s} \downarrow 0$ as $s \uparrow \infty$, such that:

$$
\lim _{y \downarrow \gamma^{s}}[m(y) / y] \geq 1
$$

We have $[M(y) / y]<1$ for all $y \in(0, \alpha)$. Consequently, for each $\gamma^{s} \in(0, \alpha)$, we have:

$$
\lim _{y \downarrow \gamma^{s}}[m(y) / y]=1
$$

Let $y^{\prime}>0$ be given. Clearly, we can find $s \in \mathbb{N}$, such that $0<\gamma^{s}<$ $\min \left(y^{\prime}, \alpha\right)$. We claim that for all $t \geq 0, y_{t}\left(y^{\prime}, \omega\right)>\gamma^{s}$ for almost every $\omega \in \Omega$. Clearly, this is true for $t=0$. We suppose this is true for $t=0, \ldots, T$. Then, using (G.4) we have $y_{T+1}\left(y^{\prime}, \omega\right)=G\left(y_{T}\left(y^{\prime}, \omega\right), \omega_{T+1}\right)>G\left(y_{T}\left(y^{\prime}, \omega\right), a\right)$ for almost every $\omega \in \Omega$. Since $y_{T}\left(y^{\prime}, \omega\right)>\gamma^{s}$ by the induction hypothesis, 
$G\left(y_{T}\left(y^{\prime}, \omega\right), a\right) \geq \lim _{y \downarrow \gamma^{s}} G(y, a)=\gamma^{s}$, since $G$ is non-decreasing in $y$. Thus, we have $y_{T+1}(y, \omega)>\gamma^{s}$ for almost every $\omega \in \Omega$. This proves our claim by induction, and shows that $\gamma^{s}$ is a safe standard of conservation. Since $y^{\prime}>0$

was arbitrarily chosen, we have shown that global conservation holds in case (ii) (b).

\section{Proof of Proposition 4:}

For any initial stock $y>0$ and realization $\omega \in \Omega$,

$$
\begin{aligned}
y_{t+1}(y, \omega) & =G\left(y_{t}(y, \omega), r_{t+1}(\omega)\right) \\
& =\left\{\frac{G\left(y_{t}(y, \omega), r_{t+1}(\omega)\right)}{y_{t}(y, \omega)}\right\} y_{t}(y, \omega) \\
& \leq \lambda\left(r_{t+1}(\omega)\right) y_{t}(y, \omega)
\end{aligned}
$$

so that by iteration, we get:

$$
y_{t+1}(y, \omega) \leq\left\{\prod_{j=1}^{t+1} \lambda\left(r_{j}(\omega)\right)\right\} y_{0}
$$

Taking log on both sides, and time-averaging, we obtain:

$$
\frac{1}{t+1} \ln y_{t+1}(y, \omega) \leq\left\{\frac{1}{t+1} \sum_{j=1}^{t+1} \ln \left[\lambda\left(r_{j}(\omega)\right)\right]\right\}+\frac{1}{t+1} \ln y_{0}
$$

Since $\left\{r_{t}(\omega)\right\}$ are iid random variables, and $\zeta \equiv E[\ln (\lambda(r))]<0$, by the strong law of large numbers, we have:

$$
P\left\{\omega \in \Omega: \lim _{t \rightarrow \infty}\left\{\frac{1}{t+1} \sum_{j=1}^{t+1} \ln \left[\lambda\left(r_{j}(\omega)\right)\right]\right\}=\zeta\right\}=1
$$

Note that $[1 /(t+1)] \ln y_{0} \longrightarrow 0$ as $t \longrightarrow \infty$. Thus, using (5.21) and (5.22), we get:

$$
\begin{aligned}
& P\{\omega \in \Omega: \text { there exists } T(\omega)<+\infty, \text { such that } \\
& \left.[1 /(t+1)] \ln y_{t+1}(y, \omega)<(\zeta / 2) \text { for all } t \geq T(\omega)\right\}=1
\end{aligned}
$$

This implies:

$$
P\left\{\omega: \lim _{t \rightarrow \infty}\left[\ln y_{t+1}(y, \omega)\right]=-\infty\right\}=1
$$

which can be rewritten as: $P\left\{\omega: \lim _{t \rightarrow \infty}\left[y_{t}(y, \omega)\right]=0\right\}=1$. 


\section{Proof of Lemma 3:}

Suppose that for some $y>0$, we have $X_{m}(y)=0$. Consider an alternative policy from $y$, where $\varepsilon \in(0, y)$ is invested, $(y-\varepsilon)$ is consumed in the initial period, and the entire output $f(\varepsilon, r)>0$ is consumed in the next period. From the definition of optimality, we must have:

$$
\begin{aligned}
0 & \leq[u(y)+\delta u(0)]-[u(y-\varepsilon)+\delta E u(f(\varepsilon, r))] \\
& =\varepsilon\left\{\frac{[u(y)-u(y-\varepsilon)]}{\varepsilon}-\frac{\delta[E u(f(\varepsilon, r))-u(0)]}{\varepsilon}\right\} \\
& =\varepsilon\left\{\frac{[u(y)-u(y-\varepsilon)]}{\varepsilon}-E\left[\left\{\frac{[u(f(\varepsilon, r))-u(0)]}{f(\varepsilon, r)}\right\}\left\{\frac{\delta f(\varepsilon, r)}{\varepsilon}\right\}\right]\right\}(5.2
\end{aligned}
$$

However, given condition (3.3), the last line in (5.25) is clearly negative, a contradiction.

\section{Proof of Proposition 6:}

Since $u^{\prime}(f(x, a)-x) \leq 0$, we have $X_{m}(f(x, a)) \geq x$, so that $m(f(x, a))=$ $f\left(X_{m}(f(x, a)), a\right) \geq f(x, a)$. The result now follows from Proposition 1.

\section{Proof of Proposition 7:}

Recall the definitions of $\hat{x}(r)$ and $\hat{x}$ in Section 3, and define a modified production function $F(x, r)$ as follows:

$$
F(x, r)=\left\{\begin{array}{lll}
{[f(\hat{x}(r), r) / \hat{x}(r)] x} & \text { for } & x \in[0, \hat{x}(r)) \\
f(x, r) & \text { for } \quad x \geq \hat{x}(r)
\end{array}\right.
$$

Clearly, $F(x, r)$ is concave in $x$ for all $r$, and $F$ is identical to $f$ for $x \geq \hat{x}$.

To prove the proposition, it suffices to confine attention to the modified dynamic optimization problem in which $F$ replaces $f$, and to show that there is a safe standard in that modified problem, which lies above $f(\hat{x}, a)=F(\hat{x}, a)$.

For the modified problem, let $W$ denote the value function, and $\chi$ the optimal investment policy correspondence. ${ }^{17}$ Define $\chi_{m}(y)=\min \{x: x \in$ $\chi(y)\}$. Since $F(x, r)$ is concave in $x$, it is easy to show that $W(y)$ is concave in $y$. Denote the right hand derivative of $W$ at any $y>0$ by $W_{+}^{\prime}(y)$. If $c$ is an optimal consumption from $y$ in the modified problem, then it can be shown ${ }^{18}$ that:

$$
W_{+}^{\prime}(y) \geq u^{\prime}(c)
$$

\footnotetext{
${ }^{17}$ In our framework, $u$ is not assumed to be strictly concave, and $F(x, r)$ is not strictly concave when $\hat{x}>0$. Thus, there need not be a unique solution to the maximization problem on the right hand side of the functional equation of dynamic programming.

${ }^{18}$ See, for example, Mirman and Zilcha (1975).
} 
and if $c>0$, then $W$ is differentiable at $y$, with:

$$
W^{\prime}(y)=u^{\prime}(c)
$$

Let $x \in(\hat{x}, \bar{x})$, and define $y^{\prime}=F(x, a)=f(x, a)$, and $x^{\prime}=\chi_{m}\left(y^{\prime}\right)$. We will now show that $x^{\prime} \geq x$, so that the stock $y^{\prime}=f(x, a)$ is a safe standard of conservation in the modified problem. Suppose, on the contrary that $x^{\prime}<x$. Since $x^{\prime}$ is an optimal investment from stock $y^{\prime}=f(x, a)$, it must be the case that $u^{\prime}\left(f(x, a)-x^{\prime}\right) \geq 0$, so that $x^{\prime} \geq \max \{f(x, a)-\xi, 0\}$. Thus, we get:

$$
x>x^{\prime} \geq \max \{f(x, a)-\xi, 0\}
$$

Observe now that $y^{\prime}-x^{\prime}=f(x, a)-x^{\prime}>0$ and therefore the principle of optimality yields for sufficiently small $\varepsilon>0$,

$$
u\left(y^{\prime}-x^{\prime}\right)-u\left(y^{\prime}-x^{\prime}-\varepsilon\right) \geq \delta E\left[W\left(F\left(x^{\prime}+\varepsilon, r\right)\right)-W\left(F\left(x^{\prime}, r\right)\right)\right]
$$

Using Fatou's lemma, this yields:

$$
\begin{aligned}
& u^{\prime}\left(y^{\prime}-x^{\prime}\right) \\
\geq & \liminf _{\varepsilon \downarrow \mathbf{0}} \delta E\left\{\left[\frac{W\left(F\left(x^{\prime}+\varepsilon, r\right)\right)-W\left(F\left(x^{\prime}, r\right)\right)}{F\left(x^{\prime}+\varepsilon, r\right)-F\left(x^{\prime}, r\right)}\right]\left[\frac{F\left(x^{\prime}+\varepsilon, r\right)-F\left(x^{\prime}, r\right)}{\varepsilon}\right]\right\} \\
\geq & \delta E\left\{\liminf _{\varepsilon \downarrow \mathbf{0}}\left[\frac{W\left(F\left(x^{\prime}+\varepsilon, r\right)\right)-W\left(F\left(x^{\prime}, r\right)\right)}{F\left(x^{\prime}+\varepsilon, r\right)-F\left(x^{\prime}, r\right)}\right]\left[\frac{F\left(x^{\prime}+\varepsilon, r\right)-F\left(x^{\prime}, r\right)}{\varepsilon}\right]\right\} \\
= & \delta E\left\{W_{+}^{\prime}\left(F\left(x^{\prime}, r\right)\right) F^{\prime}\left(x^{\prime}, r\right)\right\}
\end{aligned}
$$

Since $x^{\prime}<x$, we get:

$$
\begin{aligned}
& \delta E\left\{W_{+}^{\prime}\left(F\left(x^{\prime}, r\right)\right) F^{\prime}\left(x^{\prime}, r\right)\right\} \\
\geq & \delta E\left\{W_{+}^{\prime}(F(x, r)) F^{\prime}(x, r)\right\} \\
\geq & \delta E\left\{u^{\prime}\left(F(x, r)-\chi_{m}(F(x, r)) F^{\prime}(x, r)\right\}\right. \\
\geq & \delta E\left\{u^{\prime}\left(F(x, r)-x^{\prime}\right) F^{\prime}(x, r)\right\}
\end{aligned}
$$

the last inequality in (5.28) following from the fact that $\chi_{m}(F(x, r)) \geq x^{\prime}=$ $\chi_{m}(F(x, a))$. Since $x>\hat{x}$, we have $F(x, r)=f(x, r)$ and $F^{\prime}(x, r)=f^{\prime}(x, r)$. Thus, (5.27) and (5.28) yield:

$$
u^{\prime}\left(f(x, a)-x^{\prime}\right) \geq \delta E\left\{u^{\prime}\left(f(x, r)-x^{\prime}\right) f^{\prime}(x, r)\right\}
$$

But this contradicts condition (4.4) of the Proposition, given (5.26). 
Thus, we have established that $x^{\prime}=\chi_{m}(f(x, a)) \geq x$. That is, in the modified dynamic optimization problem, the stochastic process of optimal investments generated by $\chi_{m}$ starting from any initial stock $y \geq f(x, a)$ is bounded below by $x$ for almost every $\omega \in \Omega$, and the optimal stocks are bounded below by $f(x, a)$ for almost every $\omega \in \Omega$.

Note that for $y \geq f(x, a)$, the path generated by $\chi_{m}$ is feasible in the original (non-convex) dynamic optimization problem, which has a smaller feasible set than the modified problem. Thus, this path must be optimal in the original dynamic optimization problem. Thus, $f(x, a)$ is a safe standard of conservation for the original problem.

\section{Proof of Proposition 8:}

We will show that there exists $\eta>0$ such that $m(y)=f\left(X_{m}(y), a\right) \geq y$ for all $y \in(0, \eta)$. This will establish the result, by an appeal to Proposition 2 .

Suppose on the contrary that there exist sequences $\left\{x_{n}\right\}$ and $\left\{y_{n}\right\}$, with $x_{n} \downarrow 0$ and $y_{n} \downarrow 0$ as $n \rightarrow \infty$, such that:

$$
f\left(x_{n}, a\right)<y_{n} \text { and } x_{n}=X_{m}\left(y_{n}\right) \text { for } n \geq 1
$$

Then, we have:

$$
u^{\prime}\left(y_{n}-x_{n}\right) \leq u^{\prime}\left(f\left(x_{n}, a\right)-x_{n}\right) \text { for } n \geq 1
$$

Since $0<x_{n}<y_{n}$ for $n \geq 1$, the Ramsey-Euler equation (Lemma 4) yields:

$$
\begin{aligned}
u^{\prime}\left(y_{n}-x_{n}\right) & =\delta E\left\{u^{\prime}\left(f\left(x_{n}, r\right)-X_{m}\left(f\left(x_{n}, r\right)\right) f^{\prime}\left(x_{n}, r\right)\right\}\right. \\
& \geq \delta E\left\{u^{\prime}\left(f\left(x_{n}, r\right)\right) f^{\prime}\left(x_{n}, r\right)\right\}
\end{aligned}
$$

Combining (5.30) and (5.31), we get:

$$
\delta E\left[\left\{\frac{u^{\prime}\left(f\left(x_{n}, r\right)\right)}{u^{\prime}\left(f\left(x_{n}, a\right)-x_{n}\right)}\right\} f^{\prime}\left(x_{n}, r\right)\right] \leq 1
$$

Letting $n \rightarrow \infty$ in (5.32), we contradict condition (4.5) of the Proposition.

Proof of Proposition 9:

It is sufficient to show that:

$$
\sup \left\{\left(f\left(X^{M}(y), a\right) / y: y \in[h, \bar{x}]\right\}<1 \text { for all } h>0\right.
$$


Suppose, contrary to (5.33) that there is some $h>0$ such that:

$$
\sup \left\{\left(f\left(X^{M}(y), a\right) / y: y \in[h, \bar{x}]\right\} \geq 1\right.
$$

Then, by Proposition 1, it follows that there is $y^{*}>0$ such that under the policy generated by the optimal investment function $X^{M}$, starting from any initial stock $y \in\left(y^{*}, \bar{x}\right]$, the optimal stocks $\left\{y_{t}(y, \omega)\right\}$ are bounded below by $y^{*}$ for almost every $\omega \in \Omega$. It also implies that $X^{M}(y)>0$ for all $y \in(0, \bar{x}]$. Define:

$$
\tilde{c}=\inf \left\{\left(y-X^{M}(y)\right): y \in\left[y^{*}, \bar{x}\right]\right\}
$$

Using the upper hemi-continuity of the optimal investment correspondence $X(y)$, and the fact that $X^{M}(y)<y$ for $y \in(0, \bar{x}]$, it can be shown ${ }^{19}$ that (i) $\tilde{c}>0$, (ii) there exists $\hat{y} \in\left[y^{*}, \bar{x}\right]$ such that $\tilde{c}$ is the optimal consumption from stock $\hat{y}$; that is,

$$
\hat{y}-X^{M}(\hat{y})=\tilde{c}
$$

and (iii) from any initial stock $y \in\left[y^{*}, \bar{x}\right]$, for $t \geq 1$,

$$
c_{t}(y, \omega)=y_{t}(y, \omega)-X^{M}\left(y_{t}(y, \omega)\right) \geq \tilde{c}>0 \text { for a.e. } \omega \in \Omega
$$

Given the interiority of the optimal policy generated by $X^{M}$ from $y \in$ $\left[y^{*}, \bar{x}\right]$, Lemma 4 yields:

$$
u^{\prime}\left(\hat{y}-X^{M}(\hat{y})\right)=\delta E\left\{u^{\prime}\left(y_{1}(y, \omega)-X^{M}\left(y_{1}(y, \omega)\right)\right) f^{\prime}\left(X^{M}(\hat{y}), \omega_{1}\right)\right\}
$$

Using (5.35) and (5.36) in (5.37), we obtain:

$$
u^{\prime}(\tilde{c}) \leq u^{\prime}(\tilde{c}) \delta E\left\{f^{\prime}\left(X^{M}(\hat{y}), \omega_{1}\right)\right\}
$$

which contradicts the fact that $\delta E\left\{f^{\prime}\left(X^{M}(\hat{y}), \omega_{1}\right)\right\}<1$.

\footnotetext{
${ }^{19}$ See, for example, the proof of Lemma $2 \mathrm{~A}$, a part of the proof of Theorem 5 , in Majumdar, Mitra and Nyarko (1989).
} 


\section{References}

[1] Alvarez, L.H.R. (1998): "Optimal Harvesting under Stochastic Fluctuations and Critical Depensation", Mathematical Biosciences 152, 63-85.

[2] Alvarez, L.H.R and L. Shepp (1998): "Optimal Harvesting of Stochastically Fluctuating Populations”, Journal of Mathematical Biology 37, $155-77$.

[3] Athreya, K.B. (2002): "Stationary Measures for Some Markov Chain Models in Ecology and Economics", Technical Report, School of ORIE, Cornell University (forthcoming, Economic Theory).

[4] Boylan, E.S. (1976): "On Properties of Steady State Measures for OneSector Growth Models", International Economic Review 17, 783-85.

[5] Boylan, E.S. (1979): "On the Avoidance of Extinction in One-Sector Growth Models", Journal of Economic Theory 20, 276-79.

[6] Brock, W.A. and L.J. Mirman (1972): "Optimal Economic Growth and Uncertainty: The Discounted Case", Journal of Economic Theory 4, 479-513.

[7] Chung, K.L. (1974): A Course in Probability Theory, Second Edition, Academic Press, New York.

[8] Clark, C.W. (1973): "Profit Maximization and the Extinction of Animal Species", Journal of Political Economy 81, 950-61.

[9] Clark, C.W. (1990): Mathematical Bioeconomics: The Optimal Management of Renewable Resources, Second Edition, John Wiley \& Sons.

[10] Cropper, M. (1988): "A Note on the Extinction of Renewable Resources", Journal of Environmental Economics and Management 15, 64-70.

[11] Dechert, W.D. and K. Nishimura (1983): "A Complete Characterization of Optimal Growth Paths in an Aggregated Model with Nonconcave Production Function", Journal of Economic Theory 31, 332-54. 
[12] Hopenhayn, H.A. and E.C. Prescott (1992): "Stochastic Monotonicity and Stationary Distributions for Dynamic Economies", Econometrica 60, 1387-1406.

[13] Lande, R., S. Engen and B.-E. Saether (1994), "Optimal Harvesting, Economic Discounting and Extinction Risk in Fluctuating Populations", Nature 372, 88-90.

[14] Lewis, T.R. (1981): Optimal Resource Management under Conditions of Uncertainty, University of Washington Press.

[15] Lewis, T.R. and R. Schmalensee (1979): "Nonconvexity and Optimal Harvesting Strategies for Renewable Resources", Canadian Journal of Economics 12, 677-91.

[16] Majumdar, M. and T. Mitra (1982): "Intertemporal Allocation with a Nonconvex Technology", Journal of Economic Theory 27, 101-36.

[17] Majumdar, M. and T. Mitra (1983): "Dynamic Optimization with a Non-Convex Technology: The Case of a Linear Objective Function", Review of Economic Studies 50, 143-151.

[18] Majumdar, M., T. Mitra and Y. Nyarko (1989): "Dynamic Optimization under Uncertainty: Nonconvex Feasible Set" in G. Feiwel (ed.) Joan Robinson and Modern Economic Theory, Macmillan, 545-90.

[19] Mirman, L.J.(1972): "On the Existence of Steady State Measures for One-Sector Growth Models", International Economic Review 13, 27186.

[20] Mirman, L.J. and D. Spulber (1984): "Uncertainty and Markets for Renewable Resources", Journal of Economic Dynamics and Control 8, 239-64.

[21] Mirman, L.J. and I. Zilcha (1975): "On Optimal Growth under Uncertainty", Journal of Economic Theory 11, 329-39.

[22] Mirman, L.J. and I. Zilcha (1976), "Unbounded Shadow Prices for Optimal Stochastic Growth Models", International Economic Review 17, 121-32. 
[23] Olson, L.J. and S. Roy (2000), "Dynamic Efficiency of Conservation of Renewable Resources under Uncertainty", Journal of Economic Theory 95, 186-214.

[24] Reed, W.J. (1974): "A Stochastic Model for the Economic Management of a Renewable Resource", Mathematical Biosciences 22, 313-37.

[25] Strauch, R.E. (1966): "Negative Dynamic Programming", The Annals of Mathematical Statistics 37, 871-90. 